\title{
Performance Demonstration Program Plan for Nondestructive Assay of Drummed Wastes for the TRU Waste Characterization Program
}

Revision 2

April 2009

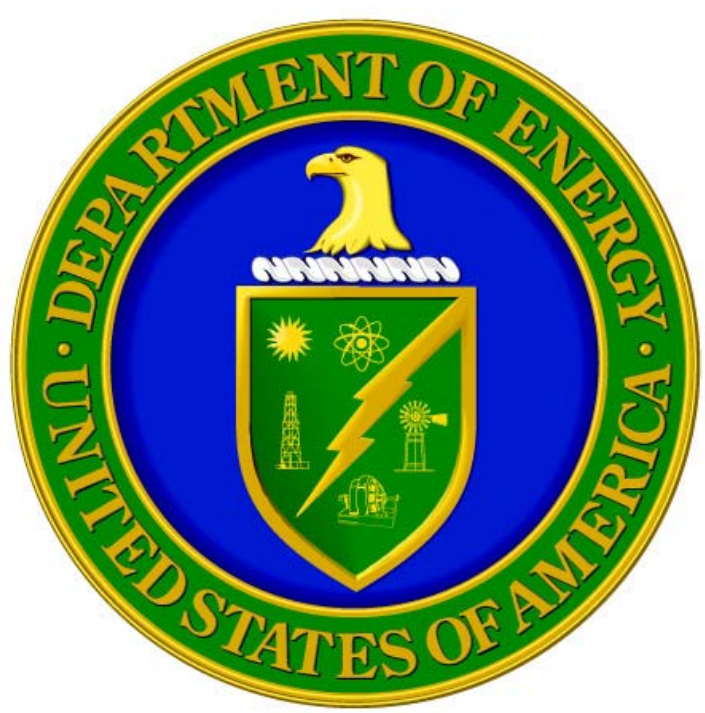

This document supersedes Revision 1 of DOE/CBF O-01-1005

\section{U.S. Department of Energy}

Carlsbad Field Office

Office of the National TRU Program 
This document has been submitted as required to:

Office of Scientific and Technical Information

PO Box 62

Oak Ridge, TN 37831

(865) 576-1188

Additional information about this document may be obtained by calling the WIPP Information Center at 1-800-336-9477. Copies may be obtained by contacting the National Technical Information Service, U.S. Department of Commerce, 5285 Port Royal Road, Springfield, VA 22101. 


\title{
Performance Demonstration Program Plan for Nondestructive Assay of Drummed Wastes for the TRU Waste Characterization Program
}

\author{
DOE/CBFO-01-1005 \\ Revision 2
}

April 2009

Approved By: //signature on file//

Date: 31 March 2009

Office Director

Office of the National TRU Program

Concurred By: //signature on file//

Date: 31 March 2009

Office Director

Office of Quality Assurance

Prepared By: //signature on file//

Date: 27 March 2009

PDP Appointee

Office of the National TRU Program 


\section{TABLE OF CONTENTS}

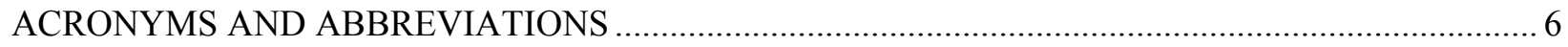

Significant Changes to the NDA Drum PDP Plan, Revision 2A ……................................................ 7

1.0 INTRODUCTION To the Performance Demonstration Program ................................................. 8

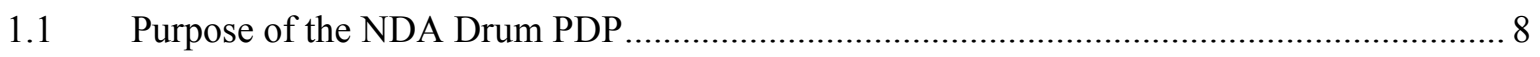

1.2 Scope and Frequency of the NDA Drum PDP ….......................................................... 9

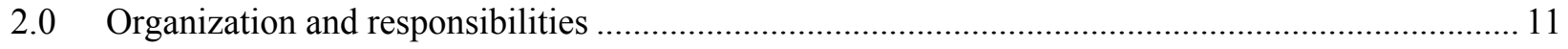

$2.1 \quad$ CBFO

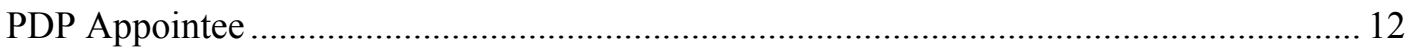

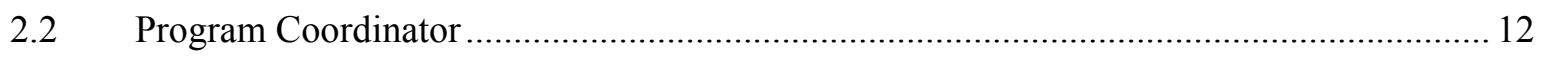

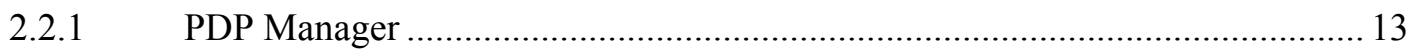

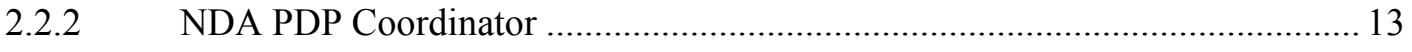

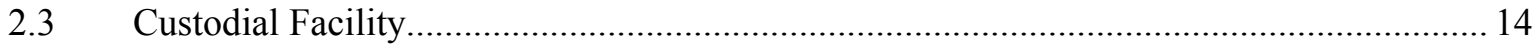

2.3.1 Custodial Facility Assay Coordinator ….................................................... 15

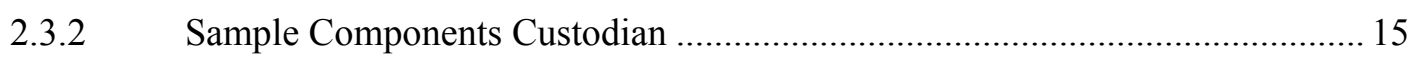

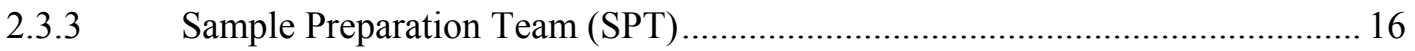

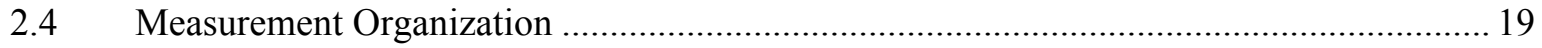

Measurement Organization Assay Coordinator ….............................................................. 19

3.0 EVALUATION OF PERFORMANCE DATA ….................................................................. 22

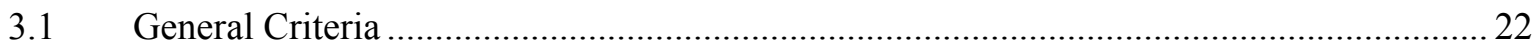

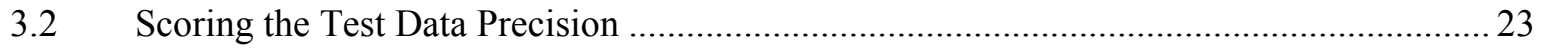

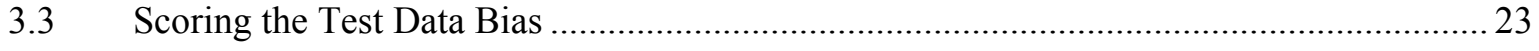

4.0 WIPP QUALIFICATION BASED ON NDA PDP TEST PERFORMANCE .............................. 24

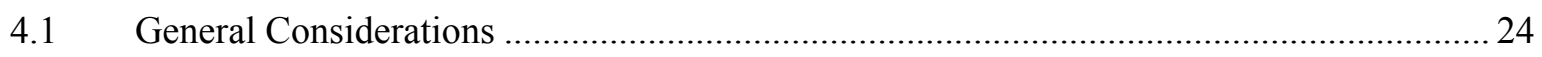

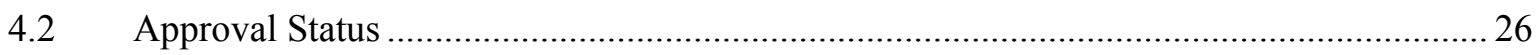

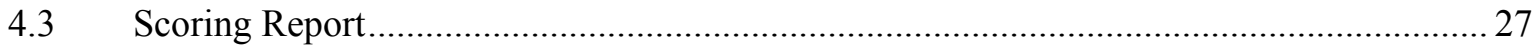

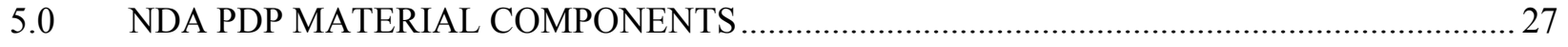

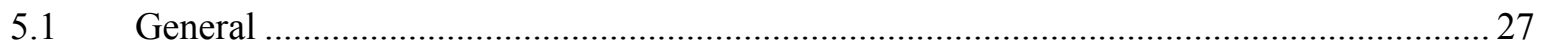

5.2 NTP Working Reference Material (WRM) Standards of the NDA PDP .......................... 28

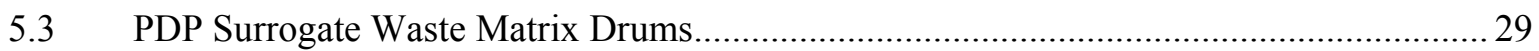

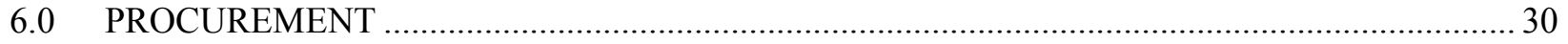

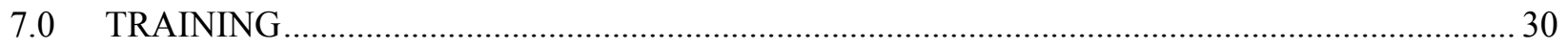

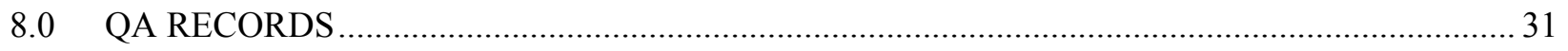

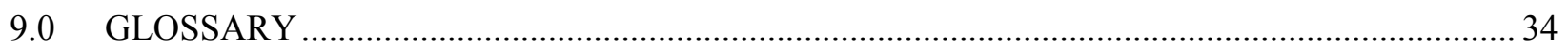

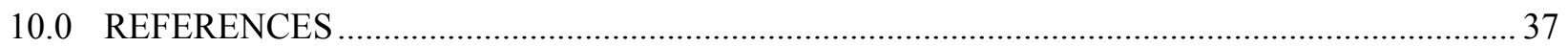


Appendix A NDA Performance Demonstration Program Forms ….................................................... 38

Appendix B Shipment and Receipt of NDA PDP Materials................................................................ 44

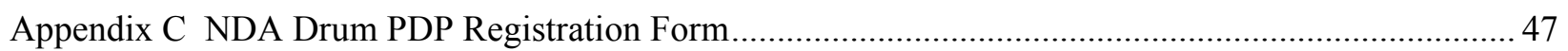

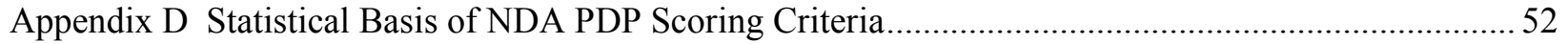

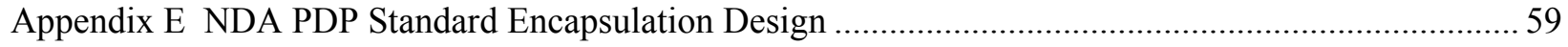

Appendix F NDA PDP Matrix Drum Specifications and Descriptions ................................................ 63 


\section{ACRONYMS AND ABBREVIATIONS}

\begin{tabular}{|c|c|}
\hline$\% \mathrm{R}$ & percent recovery \\
\hline$\%$ RSD & percent relative standard deviation \\
\hline ASNM & approval status notification memorandum \\
\hline $\mathrm{CBFO}$ & Carlsbad Field Office \\
\hline CFAC & custodial facility assay coordinator \\
\hline DOE & U.S. Department of Energy \\
\hline EA & expert analyst \\
\hline FGE & fissile gram equivalent \\
\hline $\mathrm{MDC}$ & minimum detectable concentration \\
\hline MOAC & measurement organization assay coordinator \\
\hline MP & management procedure \\
\hline NDA & nondestructive assay \\
\hline NTP & Office of the National TRU Program \\
\hline PDP & Performance Demonstration Program \\
\hline QA & quality assurance \\
\hline QAPD & Quality Assurance Program Document \\
\hline SNM & special nuclear material \\
\hline SOP & standard operating procedure \\
\hline SPT & sample preparation team \\
\hline SWB & standard waste box \\
\hline TID & tamper indicating device \\
\hline TRU & transuranic \\
\hline TWBIR & Transuranic Waste Baseline Inventory Report \\
\hline WAC & Waste Acceptance Criteria \\
\hline WG Pu & weapons grade plutonium \\
\hline WIPP & Waste Isolation Pilot Plant \\
\hline
\end{tabular}




\section{SIGNIFICANT CHANGES TO THE NDA DRUM PDP PLAN, REVISION 2A}

This document supersedes Revision 1 of the Performance Demonstration Program Plan for Nondestructive Assay of Drummed Wastes for the TRU Waste Characterization Program (DOE/CBFO-01-1005), and is a complete rewrite of that document. This revision constitutes a complete revision of each page; therefore, no change bars are included.

The document was rewritten to:

1. Reorganize the format to emphasize and consolidate cycle participant responsibilities.

2. Simplify and clarify the scoring section (section 6).

3. Clarify the explanation of the derivation of scoring criteria (Appendix D).

4. Incorporate changes in the $\mathrm{CBFO}$ organizational chart.

5. Better define the process to address failures of results from the tested NDA systems to meet NDA PDP criteria.

6. Single out the non-interfering matrix drum as distinct from the other matrix drums and to define its use for specialized circumstances.

7. Place information that is not relevant to normal NDA PDP cycle activities in the appendices. 


\subsection{INTRODUCTION TO THE PERFORMANCE DEMONSTRATION PROGRAM}

Each testing and analytical facility performing waste characterization activities for the Waste Isolation Pilot Plant (WIPP) participates in the Performance Demonstration Program (PDP) to comply with the Transuranic Waste Acceptance Criteria for the Waste Isolation Pilot Plant (WAC) (DOE/WIPP-02-3122) and the Quality Assurance Program Document (QAPD) (CBFO94-1012). The PDP serves as a quality control check for data generated in the characterization of waste destined for WIPP. Single blind audit samples are prepared and distributed to each of the facilities participating in the PDP. The PDP evaluates analyses of simulated headspace gases, constituents of the Resource Conservation and Recovery Act (RCRA), and transuranic (TRU) radionuclides using nondestructive assay (NDA) techniques.

The Carlsbad Field Office (CBFO) Office of the National TRU Program (NTP) is the review and approval authority for all PDP activities. The CBFO oversees the PDP as described in the current revisions of the following plans:

- DOE/CBFO-01-1005, Performance Demonstration Program Plan for Nondestructive Assay of Drummed Wastes for the TRU Waste Characterization Program (NDA Drum PDP Plan)

- DOE/CBFO-01-1006, Performance Demonstration Program Plan for Nondestructive Assay of Boxed Wastes for the TRU Waste Characterization Program (NDA Box PDP Plan)

- DOE/CBFO-95-1076, Performance Demonstration Program Plan for Analysis of Simulated Headspace Gases (HSG PDP Plan)

- DOE/CBFO-95-1077, Performance Demonstration Program Plan for RCRA Constituent Analysis of Solidified Wastes (RCRA PDP Plan)

This plan, the NDA Drum PDP Plan, specifically addresses the purpose, design, management, coordination, and conduct of the NDA PDP that applies to contact-handled TRU (CH-TRU) waste packaged in 208-liter (55-gallon) drums.

\subsection{Purpose of the NDA Drum PDP}

The NDA Drum PDP is designed to test participating measurement organizations' NDA systems (neutron and gamma radiation detection and analysis systems) by evaluating TRU alpha-emitting radionuclide data from NDA measurements of simulated CH-TRU waste. This plan does not address calorimetry as another NDA technique utilized for WIPP waste characterization. The relevant $\mathrm{CH}-\mathrm{TRU}$ wastes addressed by this plan are those generated and packaged in standard 208-liter (55-gallon) waste drums within the U. S. Department of Energy (DOE) complex. The NDA PDP tests provide a mechanism for the independent and objective assessment of NDA system performance and capability relative to the radiological characterization objectives and criteria of the NTP.

The nuclear material type, mass, and associated alpha activity of the NDA PDP radioactive standards have been specified and fabricated to allow assembly of NDA Drum PDP samples that 
simulate TRU alpha activity concentrations, radionuclide/isotope distributions, and physical forms typical of the DOE TRU waste inventory. The PDP waste matrix types were derived from an evaluation of information contained in the Transuranic Waste Baseline Inventory Report (TWBIR) (DOE/CAO-95-1121) to ensure representation of prevalent waste types and their associated matrix characteristics in NDA PDP testing.

In addition to the NDA PDP, the CBFO approval process includes technical evaluation of the NDA measurement systems through method and performance data supplied by the measurement organization and independent technical quality assurance audits. Qualified drum radioassay required by the WIPP may only be performed by measurement organizations and systems that have successfully participated in the NDA Drum PDP to meet performance criteria as set forth in this NDA Drum PDP Plan, have passed an audit, and are certified by CBFO.

\subsection{Scope and Frequency of the NDA Drum PDP}

The CBFO uses the NDA PDP as part of the assessment and approval process for measurement organizations and specific NDA systems characterizing waste for the NTP. Measurement organizations are required to analyze the NDA PDP drum samples using the same procedures approved and implemented for routine operational WIPP waste characterization activities. The NDA PDP is not designed for testing method or procedural changes unless those changes have been accepted by CBFO based upon approved protocol for demonstration of the method validity for the targeted wastes. The NDA PDP is designed to annually "spot check" capabilities for radioassay of two or three specific combinations of matrix type/activity type/activity level/activity distribution of typical drummed waste being assayed at a specific facility. Thus, the NDA PDP provides insufficient rigor for testing as a method validation program.

This NDA Drum PDP Plan describes the elements comprising the test program, including test apparatus, test sample configuration, and required sample analyses. The Plan further identifies and defines the responsibilities of the program entities, including the NDA PDP Coordinator, custodial facility, the facility assay coordinator, the sample preparation team (SPT), and the measurement organization.

Acceptable performance in the NDA Drum PDP must be demonstrated initially by all participating facility measurement organizations and NDA systems before the analyses of WIPP drummed wastes can be certified for shipment of the waste to WIPP. A system that has not yet successfully completed the NDA PDP testing and subsequent CBFO approval may choose to proceed at risk with analyses. The organization should recognize that data obtained prior to CBFO approval of the measurement organization, NDA systems, and procedures may be found to be unacceptable to $\mathrm{CBFO}$.

After the initial PDP approval process, each measurement organization and NDA system shall be evaluated annually, as specified in the WAC. The primary cycle for NDA Drum PDP participation will be conducted annually (i.e., every 12 months, with a one-month grace period).

In addition to the primary test cycle, the NDA PDP Coordinator may schedule a supplemental cycle prior to the next annual cycle. A supplemental NDA PDP cycle can be requested by measurement organizations for systems that have failed one or more NDA PDP samples, for newly implemented systems, or for systems that have been inactive or have undergone changes that could impact the NDA PDP approval status. Requests for a supplemental cycle must be 
submitted in writing to the CBFO. The CBFO will consider impacts on the overall WIPP schedule and program costs, and may discuss the request with the potential participants. Timing and selection of measurement systems for participation in supplemental cycles will be at the discretion of the CBFO. The CBFO will typically require the costs associated with administration of supplemental cycles to be borne by the requesting organization via funds transfer to the PDP Program Coordinator.

\subsection{NDA Drum PDP Cycle Description}

The NDA PDP cycle is a process that implements a sequence of documented activities designed to produce and evaluate NDA measurements that indicate an NDA system's capabilities to meet WIPP waste acceptance criteria.

Sequentially, the NDA PDP cycle is described by the following major activities:

- Prior to cycle initiation, the NDA PDP Coordinator identifies the facilities and measurement organizations that will be participating in the cycle, and prepares a letter documenting cycle participants for CBFO PDP Appointee signature.

- Instructions are sent to the participating facilities' Custodial Facility Assay Coordinators (CFAC) for the assembly of the cycle's PDP samples (activity type, position, and quantity). These instructions are "blind" to the measurement organization.

- The participating measurement organizations perform nondestructive radioassay of each sample utilizing the cycle-authorized NDA systems and procedures. The results are sent to the NDA PDP Coordinator.

- The NDA PDP Coordinator scores the results against criteria set forth in the NDA PDP plan.

- The NDA PDP Coordinator makes NDA system approval recommendations to the CBFO PDP Appointee based on performance of each of the participating NDA systems to NDA PDP criteria.

- The CBFO PDP Appointee combines the recommendations of the NDA PDP Coordinator with other quality assurance information gathered from onsite assessments to approve, conditionally approve, or disapprove of each of the participating NDA systems on an individual basis.

- If not approved, or if conditionally approved, a measurement organization may enter a corrective action process to obtain a higher CBFO approval status for one or more of their NDA system(s).

- A report that includes values reported by the measurement facilities, reference activity values, acceptance ranges, pass-fail status of each individual measurement system relative to the NDA PDP pass-fail criteria, and the CBFO approval status of each participating NDA system is generated by the NDA PDP Coordinator and distributed to the WIPP radioassay stakeholders. 
An NDA PDP cycle is considered initiated on the date specified in the letter transmitting the NDA PDP sample configuration instructions to the CFAC. A cycle is concluded upon issuance of the scoring report for the cycle. For the purpose of annual participation, an NDA system must participate in a PDP cycle within 12 months (plus a one-month grace period) from the date results for the cycle are received by the NDA PDP Coordinator (see section 4.2).

\subsection{ORGANIZATION AND RESPONSIBILITIES}

This section discusses the organizations involved in the NDA PDP and the responsibilities of each of these organizations. Figure 1 depicts the organizational flow of communications for the NDA PDP. Other responsibilities of the organizations involved in the NDA Drum PDP are provided in the following sections.

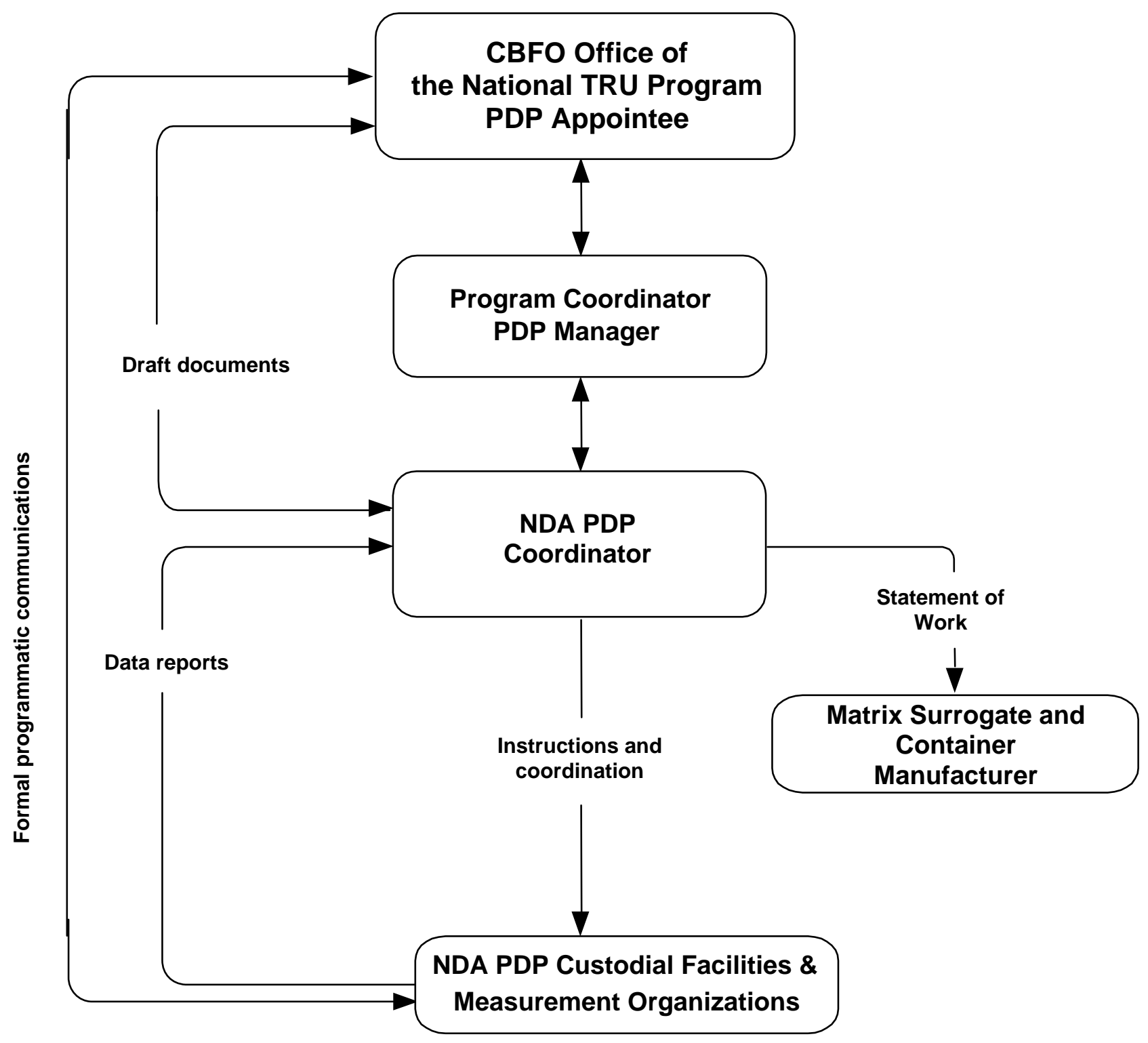

Figure 1. Organization and Communication Flowchart for the NDA PDP 


\subsection{CBFO}

The CBFO is the review and approval authority for the NDA PDP for drummed DOE waste. Programmatic direction and oversight of the PDP are performed by the NTP, the CBFO organization responsible for managing the PDP on behalf of the CBFO. The NTP designates a CBFO employee to be the PDP Appointee.

The CBFO implements the PDP by designating a Program Coordinator (currently the CBFO Technical Assistance Contractor [CTAC]), and by providing program oversight from the CBFO PDP Appointee.

The CBFO is responsible for the specification and procurement of NDA PDP standards. An NDA PDP standard is defined as a radioactive source specifically designed, prepared (or acquired), and certified for the NDA PDP. The NDA PDP standards have pedigrees traceable to nationally recognized reference bases such as the National Institute of Standards and Technology. To date, all standards used in the NDA PDP have been manufactured at the Los Alamos National Laboratory. However, sources with appropriate traceability and certification from other programs or commercial sources may be used with CBFO approval. The types of radioactive NDA PDP standards presently in the NDA PDP inventory are listed in section 5.2, Table 2.

The CBFO is also responsible for the specification and procurement of PDP matrix drums and associated components for use in the NDA Drum PDP. A PDP matrix drum is a standard 208liter (55-gallon) waste drum containing matrix materials in a configuration representative of DOE-generated wastes.

The NDA PDP is routinely assessed for utility and effectiveness by CBFO through several interrelated activities. These activities include CBFO review of the test results for each NDA PDP cycle; approval of this plan; consideration of the reports and observations of the Program Coordinator, PDP Manager, and NDA PDP Coordinator; feedback from program participants; and comments from other parties such as independent quality assurance (QA) assessors. Records of comments, decisions, or proposed actions resulting from the CBFO assessment may take any documented form including, but not limited to, routine program correspondence, meeting minutes, action items, formal review of program documents, assessment reports, and corrective actions.

\section{PDP Appointee}

The PDP Appointee is a CBFO employee designated for the oversight and individual review and approval authority for the PDP. The CBFO PDP Appointee is responsible for ensuring the implementation of each of the PDP plans by concurring with the designation of the Program Coordinator and by providing technical oversight and coordination for the program.

\subsection{Program Coordinator}

CTAC is a CBFO-designated organization that functions as the PDP Program Coordinator and technical advisor to the CBFO. The Program Coordinator designates the PDP Manager. 


\subsubsection{PDP Manager}

The PDP Manager is the individual responsible for overall management of the PDP and who ensures that all PDP responsibilities identified in the PDP Plans and those implemented through the Performance Demonstration Program Management Plan (DOE/CBFO-01-3107) and the applicable CBFO management procedures are met. The PDP Manager assigns individuals as coordinators for the three PDP elements. Each coordinator administers and coordinates their respective PDP functions such as PDP sample component preparation, subcontractor oversight, scheduling, scoring, and generating draft approval status notification memoranda for CBFO signature and PDP cycle scoring reports.

\subsubsection{NDA PDP Coordinator}

The NDA PDP Coordinator must comply with the responsibilities stipulated in this plan in accordance with the requirements of the Performance Demonstration Program Management Plan (DOE/CBFO-01-3107). The NDA PDP Coordinator is responsible for the following activities:

- Distribute and track PDP standards and matrix drums and components as appropriate to implement the program and to safeguard and secure the PDP materials.

- Maintain documentation and reconciled inventories on all NDA PDP standards and matrix drums and associated components.

- Confirm with CBFO and the prospective participants the schedule of a primary NDA PDP cycle approximately two weeks before the planned start date.

- Distribute NDA PDP sample preparation instructions and associated materials to each participating CFAC.

- Ensure training for the on-site SPT members is in accordance with the NDA PDP Plan.

- Collect and score NDA PDP sample data reports from the facility measurement organizations.

- Draft CBFO approval documentation for CBFO authorization of WIPP waste characterization by NDA systems based on their performance in an NDA PDP cycle.

- Draft CBFO approval documentation for CBFO authorization of use of (or revisions to) NDA measurement procedures prepared by the participating measurement organizations (when the new procedure or revision potentially impacts the PDP approval at the facility).

- Prepare cycle-specific scoring reports that provide NDA system-specific and NDA sample-specific scoring results for facility measurement organizations participating in a cycle.

- Ensure that records of participation and results of all NDA PDP cycles are adequately maintained in a retrievable condition.

- Review changes in the QAPD and WAC that may potentially affect this plan and provide recommendations for revising the plan as appropriate, with $\mathrm{CBFO}$ direction and approval. 
- Assist in the resolution of disputes related to NDA PDP measurement results, test sample configuration, operational restrictions, and other issues that may arise during an NDA PDP test cycle.

- Develop technical recommendations to the CBFO regarding conditional NDA characterization approvals, when warranted, based upon a system's NDA PDP performance.

- Maintain a current register of participating facility measurement organizations and their measurement system registrations. The registration documentation contains information on each assay system including a unique identity, measurement principles and modes, the NDA PDP test combinations permitted under facility measurement organization/systemspecific calibration, and administrative limits.

- Ensure the NDA PDP test material inventory at each site is sufficient to support cycle implementation.

- Initiate and orchestrate, through the Program Coordinator, the procurement of NDA PDP components to replace lost or damaged parts as necessary.

\subsection{Custodial Facility}

The custodial facility is the physical and organizational entity responsible for NDA PDP activities at the participating DOE site associated with the conduct of the NDA PDP cycles and the storage, care and proper handling of NDA PDP materials before, during, and after cycle activities. It is essential that close contact be maintained between appropriate custodial facility personnel and the NDA PDP Coordinator. The NDA PDP Participant Contact Form, found in Appendix A, must be provided to the NDA PDP Coordinator by an appropriate custodial facility manager. The form must be updated and forwarded to the NDA PDP Coordinator when contact information changes.

Each custodial facility is required to provide the NDA PDP Coordinator, in writing or by e-mail, with the name, telephone number, fax number, and address of a single contact person (CFAC) responsible for on-site administration and conduct of the NDA PDP (e.g., execution of cycle-specific schedules and instructions). The CFAC must be a full-time employee or subcontractor stationed at the facility where the NDA system(s) to be assessed is/are located. The CFAC may be a part of the SPT if he or she meets the qualifications described below. The CFAC must complete NDA Drum PDP training as indicated in section 7.0, Training.

The custodial facility is also required to provide contact information (name, telephone number, and address) for a PDP sample components custodian. The address provided must be suitable for freight and package delivery of the NDA PDP components and replacement parts. This information shall be supplied in writing or by e-mail every time the contact information changes. The PDP sample components custodian may be part of the SPT if he or she meets the qualifications described below, or may remain separate, with responsibility for the proper care and storage of non-radioactive NDA PDP components.

A two-person SPT consisting of a PDP standards custodian and a PDP standards configuration attestant shall be assigned by each custodial facility organization. The SPT members must, at a minimum, meet the following requirements: 
1. Full-time employees or subcontractors of the facility where NDA PDP materials are stored and maintained.

2. Independent of the measurement group being tested; that is, does not participate in assay measurements, reduction or analysis of the assay data, data validation activities or data reporting for the NDA PDP samples that they have prepared.

3. Trained to applicable site QA requirements in accordance with the training provided in section 7.0 of this plan.

4. Qualified to handle radioactive materials (PDP standards custodian only).

\subsubsection{Custodial Facility Assay Coordinator}

The CFAC is responsible for the following NDA PDP cycle activities throughout the year:

- Ensure timely completion of necessary and requested communication, verbal and written, with the NDA PDP Coordinator regarding all aspects of the conduct of a given NDA PDP cycle.

- Oversee and manage schedules and PDP sample transfers between the various assay systems at the site.

- Receive and distribute cycle materials and documents to the SPT in a timely manner.

- Obtain properly executed SPT documentation from the SPT and submit that documentation to the NDA PDP Coordinator within two weeks of the return of the final cycle sample to the SPT.

Note: The proper approval of NDA systems cannot be completed without the SPT documents (i.e., properly signed Sample Configuration Forms and Sample Custody Forms) being in the possession of the NDA PDP Coordinator. The completed Sample Custody Forms and Sample Configuration Forms are QA records.

- Provide notice and contact information for changes in personnel as soon as possible when changes have occurred.

- Obtain annual inventory information from the PDP sample components custodian and the PDP standards custodian for submission to the NDA PDP Coordinator each January.

\subsubsection{Sample Components Custodian}

The designated PDP sample components custodian has the following NDA PDP responsibilities:

- Ensure all NDA PDP components are properly stored and controlled to prevent unauthorized use, loss, or damage. Proper storage includes indoor, controlled climate (e.g., heated space), protection from exposure to water, and protection from physical damage (e.g., stacking on top of boxes or other drums, unnecessary exposure to forklifts). The components must be maintained in a condition consistent with that in which they were provided to the participant.

Note: PDP matrix drums are only to be used for NDA PDP purposes unless written permission has been granted by CBFO for other purposes. Questions concerning 
permissible use shall be directed to the CBFO PDP Appointee or the NDA PDP Coordinator.

- Provide the NDA PDP Coordinator, in writing or by e-mail, the inventory and location of all PDP materials (standards, drums, and parts) annually and as requested.

Note: The NDA PDP Coordinator will request the inventory from the PDP sample components custodian each January. The inventory submitted shall include a description of the condition of the PDP sample components (e.g., tamper indicating device (TID) security lanyards, matrix spacers, source insert fixtures, and locating pins). Digital images of the NDA PDP sample components can be submitted in addition to condition descriptions.

- Notify the NDA PDP Coordinator of the need for replacement or repair of any NDA PDP components. If minor repairs to drums and related materials are needed, the facility may make those repairs with NDA PDP Coordinator approval.

- Return the drum or component in accordance with instructions provided by the NDA PDP Coordinator when requested. Replacement parts will be provided by the NDA PDP Coordinator.

- Ensure proper palleting and packaging of the PDP drums and components prior to shipping (see Appendix B for information on shipping and receiving of NDA PDP drums).

\subsubsection{Sample Preparation Team (SPT)}

The SPT is a two-person team consisting of a PDP standards custodian and a PDP standards configuration attestant with the following NDA PDP responsibilities:

- Upon initiation of sample preparation for an NDA PDP cycle, examine all required PDP sample components (e.g., insert fixtures, locating pins, PDP standards and matrix spacers) identified on the PDP Sample Configuration Form (found in Appendix A) prepared by the NDA PDP Coordinator. The purpose of the pre-load examination is to determine if any components are missing or damaged.

- Notify the NDA PDP Coordinator of any PDP component loss or damage. If the component is a consumable item (such as a TID, form, insert tube, or matrix spacer), determine if a spare component is available from the site inventory. If so, replace the missing or damaged component with the spare, document the replacement as appropriate, and notify the NDA PDP Coordinator of the replacement. If there is no spare available, contact the NDA PDP Coordinator for a replacement.

- Select the proper serial-numbered matrix drum for installation of NDA PDP standards and matrix spacers. The PDP standards configuration attestant shall verify that the proper drum is selected for PDP radioactive standard loading.

- Identify the correct NDA PDP standards using the applicable PDP Sample Configuration Form (found in Appendix A). The PDP Sample Configuration Form provides the SPT with the PDP standard placement within the drum.

- Coordinate the placement of the PDP matrix drum, matrix spacers, NDA PDP standards, and related equipment (such as TIDs) into a designated sample preparation area. 
- Assemble the matrix drum sample following the instructions of the NDA PDP Sample Configuration Forms.

- Independently verify that the source positioning is correct by initialing the PDP Sample Configuration Form. If a PDP sample component is damaged, missing, or misplaced, this information must be reported to the assay coordinator, who will notify the NDA PDP Coordinator.

- Maintain the integrity of each prepared NDA PDP sample from the time it is sealed until disassembly. Other than the SPT, no observers are permitted during the PDP sample preparation process without the prior permission of the NDA PDP Coordinator. The SPT and any observers shall not divulge any information regarding the loading and configuration of the PDP matrix drums test sample until the measurement results are released by the CBFO or as indicated by the NDA PDP Coordinator.

- Prepare a PDP Sample Custody Form for Nondestructive Assay (found in Appendix A) for transfer of the sample to the facility measurement organization.

- Return any unused Drum PDP materials to storage and secure the NDA PDP standards storage area with a TID. A site-specific security system may be used in place of the supplied TID as long as the prevention of unauthorized access to the unused standards or storage-related documentation can be ensured and documented.

- Maintain all records of PDP sample preparation in strict confidence until CBFO distributes a final report, or as notified by the NDA PDP Coordinator.

- Determine the condition of the TID that seals the copy of the PDP Sample Configuration Form (found in Appendix A) attached to the drum sample when the sample is returned from the measurement organization.

- Disassemble the PDP samples as soon as possible after the return of the samples from the measurement organization.

- Submit completed SPT documents to the CFAC as soon as the last NDA PDP sample for the cycle has been properly disassembled.

The PDP standards custodian has the following specific NDA PDP responsibilities as part of the overall SPT responsibilities listed above:

- Load each insert fixture with NDA PDP standards per the PDP Sample Configuration Form and insert each loaded insert fixture into the identified position of the matrix drum insert fixture as specified on the PDP Sample Configuration Form.

- Seal the envelope containing a copy of the completed PDP Sample Configuration Form with a tamper-indicating security seal and affix it to the PDP matrix drum.

- Transfer the PDP samples and Sample Custody Forms to the measurement organization by obtaining the signature of the measurement organization's responsible individual for each prepared PDP sample.

- Retrieve the appropriate PDP Sample Custody Form for Nondestructive Assay for each PDP sample to be disassembled from the measurement facility at the time the PDP samples are returned for disassembly. 
- Determine the condition of the TID on the drum lid locking bolt that indicates tampering with the matrix drum source insert fixtures (Appendix F, Figure F-7).

- Break the TID (if intact) and remove the security lanyard, allowing the insert fixtures containing the PDP standards to be removed.

- Secure the evidence and note the condition on the Sample Custody Form if any TID, custody seal, matrix drum, or standard shows evidence of tampering. Notify the NDA PDP Coordinator through or in conjunction with the CFAC regarding this condition. The NDA Coordinator, in consultation with $\mathrm{CBFO}$, will provide written instruction as to how to proceed with a test drum that indicates tampering. Implement corrective actions as directed by the NDA PDP Coordinator.

- Verify that the source positioning is correct by initialing the PDP Sample Configuration Form. Record any discrepancies on the PDP Sample Configuration Form along with initials and date.

- Remove each PDP standard from the source insert fixture during disassembly.

- Document the disassembly as complete by signing the bottom of the Nondestructive Assay PDP Sample Custody Form and indicating "disassembled" in the disposition section of the form.

- Coordinate the return of the PDP matrix drums with the PDP sample components custodian.

- Return the PDP standards to the designated, secured storage areas using the facility's storage procedures.

- Ensure that all completed SPT documentation is sent to the CFAC as soon as possible after cycle samples have been disassembled.

- Ensure that under no circumstances any modifications are performed on the NDA PDP standards.

The PDP standards configuration attestant has the following specific NDA PDP responsibilities as part of the overall SPT responsibilities listed above:

- Verify that placement of the NDA PDP standards are correct and document the verification by initialing the specified box of the PDP Sample Configuration Form.

- Thread the security lanyard through all the insert fixture TID rings and seal the PDP sample with a serialized TID.

- Record the TID number on the Sample Configuration Form in the space provided.

- Verify that the source positioning is the same as indicated on the PDP Sample Configuration Form by initialing the form during sample disassembly. If not the same, document the discrepancy and notify the NDA PDP Coordinator and the CFAC.

- Ensure that the PDP standards custodian has completed the disassembly documentation by signing the Sample Configuration Form and indicating "disassembled" in the disposition box at the bottom of the form. 


\subsection{Measurement Organization}

The measurement organization must provide a point of contact to the NDA PDP Coordinator for all measurement and reporting activities of an NDA PDP cycle in which that organization is involved. That contact will have the designation of measurement organization assay coordinator (MOAC).

\section{Measurement Organization Assay Coordinator}

The MOAC is the point of contact with the NDA PDP Coordinator for all measurement and reporting activities of an NDA PDP cycle. The MOAC or designee has the following NDA PDP responsibilities:

- Complete an initial PDP System Registration Form, or update any existing form, for each NDA system to be tested in an upcoming NDA PDP cycle and send the form to the NDA PDP Coordinator. The form will include the system name, calibration range, operational limits, and other administrative information affecting NDA PDP testing. Appendix C contains the System Registration Form and instructions for registering a system for the NDA PDP.

- Respond to requests from the NDA PDP Coordinator regarding systems that will be participating in upcoming NDA PDP cycles.

- Accept PDP samples, ensuring adherence to chain-of-custody protocols, and confirm the accuracy of each Sample Custody Form during transfer of the PDP samples from the SPT of the custodial facility.

- Inspect the condition of the sample seals by checking the TIDs and ensuring they are intact. If a problem exists with the integrity of a TID, the MOAC or designee shall not accept the sample from the SPT member.

- Review, sign, and date the Sample Custody Form provided that the TIDs are intact and the Sample Custody Forms are properly completed. This custodial signature means that the measurement organization accepts the PDP sample for analysis.

- Document on the PDP Sample Custody Form accompanying the PDP sample all subsequent transfers of the PDP sample within the measurement group and the return of the sample to the SPT.

- Ensure that the final signature on the Sample Custody Form is made by the SPT when the PDP sample is accepted by the SPT from the measurement organization for disassembly at the conclusion of assay system measurements.

- Ensure that the facility measurement organization performs six (6) qualified measurements of each PDP sample using the assay procedures that are implemented for routine waste assays, and approved for use in the WIPP waste characterization program. These procedures must have been reviewed and approved by CBFO.

Note: A qualified measurement is one that would be determined by the NDA operator to meet the specifications of the approved procedure and would thereby be accepted for review by the expert analyst (EA) as if the measurement were a single measurement obtained on a WIPP-bound waste sample. It is not permissible to select six (6) qualified measurements from a larger set of qualified measurements and submit them as the NDA 
PDP cycle measurement replicate set. If, during review of results by the EA or other data reviewing personnel, a measurement or other data problem is determined, one of the following must occur: 1) A general procedure (not one specified for NDA PDP samples only) is followed, that would also be followed for actual waste sample data, to address the identified problem, or 2) the MOAC or designee will notify the NDA PDP Coordinator about the problem for further determination of how to proceed.

- Ensure the PDP sample is completely removed and replaced between each sequential measurement.

- Complete and report analyses as soon as possible, except in cases where one or more of the six (6) measurements are judged by the NDA analyst to be non-compliant as a WIPP waste qualified measurement.

- Identify instrument failure or operator error based on the methods used by the measurement organization in identifying these issues when analyzing WIPP samples.

Note: If a measurement is determined to be inadequate, contact the NDA PDP Coordinator. It is not acceptable to evaluate for measurement failure by reviewing NDA PDP data and determining the existence of precision problems. If instrument failure or operator error can be identified in accordance with approved procedures, the NDA PDP Coordinator is to be notified. Based on circumstances, the NDA PDP Coordinator may give permission to repeat one or more of the six-replicate measurements in the set prior to submitting data on the Nondestructive Assay Performance Demonstration Program Report Form (Appendix A).

- Ensure that a signed measurement report for each replicate analysis of each PDP sample is forwarded directly to the NDA PDP Coordinator. The Performance Demonstration Program Report Form - Nondestructive Assay (Appendix A) shall be used to report the data to the NDA PDP Coordinator. Continuation sheets may be used if facility measurement organization comments exceed the allotted space. Completed forms shall be signed by a facility measurement organization staff member, generally the EA. Reports should contain any other sample measurement information deemed relevant by the facility measurement organization. Corrections to data, or data inadvertently omitted from the report form, will be accepted with adequate justification, if the NDA PDP Coordinator is notified before the CBFO approval status notification memorandum (see section 4.2) is issued.

- Ensure that all analytical reports are received by the NDA PDP Coordinator within 28 calendar days after the cycle start date. The start date is provided in the cycle initiation letter sent to the CFAC by the NDA PDP Coordinator. The NDA PDP Coordinator will send a courtesy copy of the cycle initiation letter to the MOAC at the same time the letter is sent to the CFAC.

- Complete and report analyses as soon as possible following all reviews called for in routine operating procedures. Should these reviews indicate the need for data manipulation commonly allowed by the routine operating procedure that could result in a delay in reporting the results of the analyses, the MOAC should notify the NDA PDP Coordinator of the cause and duration of any delay. The notification must be followed by a written request for extension of the due date with a proposed new due date. If granted by $\mathrm{CBFO}$, the extension approval will designate a new due date. 
Note: If an extension has not been requested prior to the applicable due date (i.e., either the original due date or any subsequently granted extended due date), CBFO may elect not to receive subsequent data for that NDA PDP cycle depending upon the circumstances.

- Ensure that each NDA PDP Report Form contains, at a minimum, the following information for each determination:

- the reporting facility measurement organization

- system ID designated during the NDA PDP registration process

- PDP cycle for which the data are being reported

- NDA PDP Sample ID from the PDP Sample Custody Form for Nondestructive Assay

- measurement system and method (including software/hardware configuration version) used for each isotope (measurement systems using constant isotope ratios are to be indicated on the Report Form)

- NDA PDP sample measurement replicate number corresponding to the analytical data

- activity in curies for each isotope identified and quantified from the list in section 5.2

- total uncertainty for each identified isotope at one standard deviation

- total ${ }^{239} \mathrm{Pu}$ fissile gram equivalent (g) and associated total uncertainty at one standard deviation

- total TRU alpha activity and associated total uncertainty (curies) at one standard deviation

- thermal power and associated uncertainty at one standard deviation (W)

- elapsed counting time

- date and time of sample analysis

- the reason for no value when not reported (e.g., < minimum detectable concentration $(\mathrm{MDC}))$

- Maintain in a traceable and auditable condition all records generated by the measurement organization during the conduct of an NDA PDP cycle. All such records are QA records that must be maintained in storage conditions and for durations as required by the QAPD and other implementing QA documents and procedures.

- Review the draft CBFO approval status notification memorandum (ASNM) (see section 4.2) provided by the NDA PDP Coordinator. This review will occur prior to the NDA PDP Coordinator submitting the ASNM to CBFO for final approval, signature, and transmittal to the stakeholders. This review is to assist in ensuring that information contained in the memorandum is correct. The MOAC will:

- ensure the proper identity of the system, procedures and other relevant information provided to the NDA PDP Coordinator by the measurement organization during the cycle are referenced in the ASNM;

- request the NDA PDP Coordinator clarify the language used in the memorandum, if necessary;

- request the NDA Coordinator to seek final resolution by CBFO regarding any disapproval or conditional approval if the approval status or conditions imposed are disputed, with technical justification, by the measurement organization. 


\subsection{EVALUATION OF PERFORMANCE DATA}

\subsection{General Criteria}

In the NDA PDP, measurement system performance is evaluated using the measurement data precision and bias. Precision is defined as the percent relative standard deviation (\%RSD); the standard deviation of the six sequential replicate measurements divided by the known value times $100 \%$. Bias is the systematic error component of the total measurement uncertainty determined as the ratio of the mean of the measurement replicate set to the known value times $100 \%$, or the percent recovery $(\% \mathrm{R})$.

The precision acceptance criteria vary as a function of the activity range of the sample and whether the NDA PDP sample matrix is considered to be interfering or non-interfering. The bias acceptance range does not vary as a function of sample alpha $(\alpha)$ activity, but does vary as a function of sample matrix category (interfering or non-interfering). Categorized $\alpha$ activity ranges that apply to the NDA PDP data acceptance criteria are listed in Table 1.

The scoring system for the NDA PDP is pass-fail. In order to pass an NDA PDP test cycle criterion, the six-replicate measurement data set of a particular test sample must meet the bounds and limits listed in Table 1. See Appendix D for an explanation of the origins and statistical basis for the precision and bias acceptance criteria.

Table 1. NDA PDP activity ranges and associated scoring acceptance criteria.

\begin{tabular}{|c|c|c|c|c|c|}
\hline \multirow[b]{2}{*}{$\begin{array}{l}\text { Activity } \\
\text { range }\end{array}$} & \multirow[b]{2}{*}{$\begin{array}{c}\text { Range of sample } \\
\text { activity in } \\
\alpha \text {-curies }\end{array}$} & \multicolumn{2}{|c|}{$\begin{array}{c}\text { Maximum Measured } \\
\text { Precision }^{\mathrm{b}}\end{array}$} & \multicolumn{2}{|c|}{$\begin{array}{c}\text { Bias Range } \\
\left(\% R_{L} \text { and } \% R_{U}\right)\end{array}$} \\
\hline & & $\begin{array}{l}\text { Non- } \\
\text { interfering } \\
\text { matrix } \\
(\% \text { RSD })\end{array}$ & $\begin{array}{l}\text { Interfering } \\
\text { matrix } \\
(\% \mathrm{RSD}) \\
\end{array}$ & $\begin{array}{l}\text { Non- } \\
\text { interfering } \\
\text { matrix } \\
(\% \mathrm{R})\end{array}$ & $\begin{array}{c}\text { Interfering } \\
\text { matrix } \\
(\% \mathrm{R})\end{array}$ \\
\hline Low & $>0$ to 0.02 & 14 & 16 & $\begin{array}{l}\text { Lower: } 70 \\
\text { Upper: } 130\end{array}$ & $\begin{array}{l}\text { Lower: } 40 \\
\text { Upper: } 160\end{array}$ \\
\hline Mid-Low & $>0.02$ to 0.2 & 10.5 & 12 & $\begin{array}{l}\text { Lower: } 70 \\
\text { Upper: } 130\end{array}$ & $\begin{array}{l}\text { Lower: } 40 \\
\text { Upper: } 160\end{array}$ \\
\hline $\begin{array}{l}\text { Mid- } \\
\text { High }\end{array}$ & $>0.2$ to 2.0 & 7 & 12 & $\begin{array}{l}\text { Lower: } 70 \\
\text { Upper: } 130\end{array}$ & $\begin{array}{l}\text { Lower: } 40 \\
\text { Upper: } 160\end{array}$ \\
\hline High & $>2.0$ & 3.5 & 6 & $\begin{array}{c}\text { Lower: } 70 \\
\text { Upper: } 130\end{array}$ & $\begin{array}{c}\text { Lower: } 40 \\
\text { Upper: } 160\end{array}$ \\
\hline
\end{tabular}

a. Applicable range of TRU activity contained in a PDP sample; units are curies of alpha-emitting TRU isotopes with half-lives greater than 20 years.

b. Measured precision that must be met to satisfy the precision criteria at the $95 \%$ upper confidence bound, based on six replicates. The values are one relative standard deviation referenced to the known value for the test.

c. $\% R_{L}$ and $\% R_{U}$ values used in Equation 3 to determine the $95 \%$ confidence bound for the ratio of the mean of the measured values to the known value, expressed as a percent. 


\subsection{Scoring the Test Data Precision}

Purpose: To demonstrate compliance with the NDA PDP data acceptance criteria for precision, NDA measurement results from six replicate analyses of an NDA PDP sample of known total TRU $\alpha$-activity and known matrix category (i.e., interfering or non-interfering) are used to determine measurement system precision.

Criteria: The results reported for total TRU $\alpha$-activity from the six replicate measurements of an NDA PDP sample shall not exceed the allowable \%RSD of Table 1, column 3 for the noninterfering matrix samples, or column 4 for the interfering matrix samples.

Method: The analytical results from the six replicate measurements of an NDA PDP sample are used to calculate the \%RS:

$$
\% \mathrm{RSD}=100 \% \times \frac{1}{\mu_{0}} \sqrt{\frac{1}{n-1} \sum_{i=1}^{n}\left(x_{i}-\bar{x}\right)^{2}}
$$

where:

$$
\begin{aligned}
& x_{i}=\text { replicate sample value } \\
& n=\text { number of replicate measurements } \\
& \mu_{0}=\text { actual known PDP sample value } \\
& \bar{x}=\text { replicate sample mean, defined by } \\
& \qquad \bar{x}=\frac{1}{n} \sum_{i=1}^{n} x_{i}
\end{aligned}
$$

The measured \%RSD is compared with the applicable limits listed in Table 1 . If the \%RSD value determined from the measurement data is less than or equal to the matrix-type and total TRU $\alpha$-activity range-specific value in Table 1 , column 3 or 4 , the measurement system passes the precision test for that sample.

Actions: Any sample for which results exceed the appropriate acceptance criterion for precision will be scored as failed. The impact of failing an acceptance criterion is given in section 4.2. In accordance with section 4.2, the measurement organization assay coordinator is responsible for ensuring that appropriate corrective actions are taken, if deemed necessary by the measurement organization or, subsequently, by CBFO.

\subsection{Scoring the Test Data Bias}

Purpose: NDA results for replicate analyses for NDA PDP samples of known TRU $\alpha$-activity and known matrix category (i.e., interfering or non-interfering) are used to determine the bias associated with measurement system determinations of total TRU $\alpha$-activity. In the NDA PDP, the total bias determined using the six replicate measurement results includes components attributable to both variance and bias, including effects due to sample matrix configuration and nuclear material characteristics. 
Criteria: The results reported for total TRU $\alpha$-activity must make true Equation 3 . The selection of the appropriate criteria $\left(\% \mathrm{R}_{\mathrm{L}, \mathrm{U}}\right.$ values) specified in Table 1 is based on the matrix category (i.e., interfering or non-interfering) but does not vary with activity level.

Method: Measurement system bias shall be evaluated by calculating the sample mean of the six sequential replicate measurements of the NDA PDP sample.

For evaluation of sample-specific measurement system bias limits, the Student's $t$-distribution $t_{0.975}$ percentile value is used in conjunction with the sample mean ( $\bar{x}$ as defined in equation 2 ), the percent relative standard deviation (\%RSD of the sample replicates as defined in Equation 1$)$ and the appropriate lower and upper bounds $\left(\% \mathrm{R}_{\mathrm{L}}\right.$ and $\left.\% \mathrm{R}_{\mathrm{U}}\right)$ in columns 5 and 6 of Table 1 (as determined by matrix type). The equation that establishes the bias acceptance criteria is expressed as:

$$
\left(\% \mathrm{R}_{\mathrm{L}}+1.049 \times \% \mathrm{RSD}\right)<\left(100 \% \times \frac{\bar{x}}{\mu_{0}}\right)<\left(\% \mathrm{R}_{\mathrm{U}}-1.049 \times \% \mathrm{RSD}\right)
$$

where:

$$
\begin{aligned}
\% \mathrm{R}_{\mathrm{L}} & =\text { low percent recovery limit specified in Table } 1 \text {, column } 5 \text { or } 6 \text {, as appropriate } \\
\% \mathrm{R}_{\mathrm{U}} & =\text { upper percent recovery limit specified in Table } 1 \text {, column } 5 \text { or } 6 \text {, as appropriate } \\
\bar{x} & =\text { replicate set average (sample mean), defined in Equation } 2 \\
\mu_{0} & =\text { actual known PDP sample value }
\end{aligned}
$$

The measurement will pass the bias acceptance criterion if Equation 3 is satisfied and will fail if Equation 3 is not satisfied.

Actions: If any measurement system produces results that do not satisfy the appropriate acceptance criterion for bias, then that NDA system will be scored as failed for that sample. The impact of failing an acceptance criterion is given in section 6.3. In accordance with section 4.2, the measurement organization assay coordinator is responsible for ensuring that appropriate corrective actions are taken, if deemed necessary, initially by the measurement organization or subsequently by CBFO.

\subsection{WIPP QUALIFICATION BASED ON NDA PDP TEST PERFORMANCE}

\subsection{General Considerations}

The primary purpose of the NDA PDP is to independently acquire data from participating NDA measurement systems for the evaluation of the system and facility measurement organization's capability to produce data that meet the quality requirements for the WIPP, and to reveal any technical or quality assurance-related deficiencies that may negatively impact the characterization of WIPP wastes. Through NDA PDP evaluations, information is gathered on system performance, NDA measurement organization management quality processes, NDA procedural adequacy, effectiveness and implementation, corrective action effectiveness, and overall data quality assurance. Finally, the NDA PDP functions to provide technical justification to $\mathrm{CBFO}$ for approving (conditionally or without condition) or disapproving NDA systems, procedures, and organizations for making qualified WIPP waste NDA measurements. 
Where possible, the procedures and measurement system parameters used to analyze NDA PDP samples are to be the same as those used in the analysis of WIPP wastes. Due to the fact that NDA PDP samples are analyzed six separate times (unlike WIPP waste samples), the procedure for conducting these replicates will be unique to the NDA PDP. Because count time determinations for NDA PDP samples are directly related to the precision of the results and are scored against NDA PDP criteria, the NDA PDP sample count times must have a direct, procedural correlation with count time determinations for WIPP waste samples. Acceptable Knowledge (AK) does exist with respect to NDA PDP samples (e.g., matrix type, nominal density, and fill height). Thus, when the PDP sample AK corresponds to actual waste AK, in which count time protocol is specified or formulated, the count time protocol must also be applied to the NDA PDP samples. Any deviations from such protocol must either have a real waste sample procedural justification or specific and documented approval of CBFO as communicated in correspondence with the NDA PDP Coordinator.

The CBFO determines the approval status of a measurement organization's NDA measurement capability for any individual NDA system based on passing or failing NDA PDP scoring criteria. A passing or failing score for a given criterion of the NDA PDP is related to the characteristics of the PDP sample analyzed and is therefore subject to evaluation based upon all of the following specifics of the test: 1) the NDA system; 2) the sample matrix; 3) the activity type; 4) the activity range; and 5) the particular scored parameter (bias or precision). Thus, a pass or fail in the NDA PDP has specific, though potentially broad, technical and programmatic ramifications.

Obtaining a passing score on all criteria for all samples presented to an NDA system within an NDA PDP cycle provides CBFO with the evidence and justification for not imposing any restrictions upon WIPP measurements made by that NDA system during the approval period. Any restrictions that have not been addressed by the measurement organization through CBFOapproved corrective action, recalibration, etc., continue as restrictions upon the system through the subject cycle approval period. Approval is based upon the use of the measurement organization's CBFO-approved procedures for samples that exhibit characteristics within the approved system's various parameter calibration ranges.

A failure of an NDA system to meet one or more of the scoring criteria, however, may require the need for further investigation of the cause of that failure. It is the responsibility of the affected measurement organization, with assistance and oversight by the NDA PDP Coordinator and other CBFO technical support staff, to establish the most likely cause(s) for its failure. Once the cause is reviewed and concurred upon by $\mathrm{CBFO}$, it must be evaluated for potential effect upon WIPP waste analyses. It is incumbent upon the participating measurement organization to demonstrate through technical justification the impact that an NDA PDP criterion failure has on WIPP waste measurements. CBFO is the final authority on establishing this impact determination.

If the measurement organization can demonstrate that the cause of an NDA PDP criteria failure has no impact on routine WIPP waste analyses, no restrictions will be placed upon the system. Based on the cause of failure, CBFO may determine that multiple systems within the organization should be restricted. Restrictions may include disapproval of all measurements made by the system or may be conditional approval restricting the system from performing measurements for WIPP based on certain waste parameters such as waste types, activity types, activity levels, waste densities, measurement modes within an NDA system, or any other 
reasonable restriction that is determined through the root cause process as technically justified for prevention of unacceptable data quality for meeting the WIPP waste acceptance criteria.

As specified in section 4.2, CBFO will provide a written ASNM documenting approval, disapproval, or conditional approval. The ASNM will provide the basis for any restrictions. For conditional approvals, the ASNM will specify the restrictions that are imposed by CBFO. The measurement organization is requested to review the draft ASNM for comment and clarification prior to its finalization (see section 2.4.1).

If a system has been conditionally approved or not approved, the measurement organization may choose to take one of the following courses of action:

- Accept the conditional approval or disapproval with no further action (Note: accepting disapproval will require the measurement organization to halt the use of the system for performing qualified NDA measurements on WIPP waste until the system can be approved or conditionally approved. Likewise, accepting conditional approval limits the system to making qualified WIPP measurements only on samples meeting the specified conditions of the approval);

- Challenge the conditional approval or disapproval with additional data and other technical justifications for a change in the CBFO approval status;

- Initiate a corrective action to eliminate the causes for the condition adverse to quality that resulted in the NDA PDP failure, and, if necessary, request a supplemental cycle.

If the measurement organization chooses to initiate corrective actions, it must:

1. Identify the basis of the failure that addresses all contributing components, technical and/or administrative, and submit them in a corrective action plan (CAP).

2. Obtain CBFO approval of the CAP.

3. Implement the CBFO-approved CAP and provide evidence that the plan effectively addresses the deficiency and the actions are completed in a corrective action report CAR.

4. Obtain final CBFO approval, conditional or otherwise, to process WIPP wastes after completion of the CAR.

$\mathrm{CBFO}$ or the measurement organization may require, through the CAP, analysis or additional measurements of NDA PDP test samples as part of the verification that the corrective actions are effective.

If the CBFO determines that the measurement organization is not responsive or is not adequately addressing a condition adverse to quality that has been determined through its participation in the NDA PDP, the CBFO may formally request a corrective action.

\subsection{Approval Status}

Once the CBFO has determined an organization's measurement systems and methods status as "approved," "conditionally approved," or "not approved," such status shall remain in effect until $\mathrm{CBFO}$ is presented justification to change that status. All measurement systems must participate in the PDP cycle annually in order to remain qualified to perform WIPP analyses. Measurement systems obtaining approval status through a supplemental cycle must participate in the next 
regular primary cycle for reevaluation of their approval status. This participation could change the approval status of a system and will reset the 13-month approval period as stated below.

The approval period for a measurement system begins with the date that signed data reports from an NDA PDP cycle are received by the NDA PDP Coordinator. At the end of the 13th month, a system that has not yet successfully completed the analyses of NDA PDP samples to requalify may choose to proceed at risk with WIPP analyses, or to cease operations. The organization should recognize that data obtained at risk may be found to be unacceptable to CBFO for the WIPP. Data generated at risk cannot be used for characterizing waste for shipment to the WIPP until:

- $\quad$ The system and methods used to collect and process the data satisfy the CBFO through successful NDA PDP sample measurement performance, and

- The data obtained during the "at risk" period have been reconciled through the disposition of a nonconformance report.

CBFO approval status notification memoranda are sent to the corresponding DOE Operations Office involved, the participant measurement organization, the U.S. Environmental Protection Agency (EPA) and other relevant stakeholders as deemed appropriate by CBFO.

\subsection{Scoring Report}

The NDA PDP Coordinator shall review, evaluate, and score the reported data results for all facility measurement organizations and participating NDA systems and compile them into a scoring report. The cycle-specific scoring report is normally scheduled for delivery to CBFO within approximately four weeks after the final data are received from all participants in the cycle, including receipt of revised data as a result of corrective actions. The report shall include the values reported by the measurement facilities, the reference activity values, the acceptance ranges, the pass-fail status of each individual measurement system, and the CBFO approval status of each participating NDA system.

Copies of the scoring report are distributed to each of the DOE Operations Offices involved in the NDA PDP cycle being reported, each of the participating measurement organizations, and other individuals and organizations deemed appropriate by the CBFO. Distribution will be in an electronic format.

\subsection{NDA PDP MATERIAL COMPONENTS}

\subsection{General}

Without written CBFO permission, NDA PDP matrix drums or NTP standards are not to be used for any activity other than the performance of the NDA PDP. Written requests for any other uses must be submitted to the $\mathrm{CBFO}$ specifying each use requested or requesting an ongoing protocol for usage after approval by $\mathrm{CBFO}$. CBFO will provide written approval or disapproval of the request. 
Under no circumstances are the NDA PDP standards to be used for measurement system calibration. Under no circumstances are any modifications to be performed on the NDA PDP standards (see section 2.3.3 for custodial facility responsibilities regarding the NDA PDP standards).

The custodial facility is responsible for assigning a secure storage area for all NDA PDP components and meeting applicable safeguards, security, and safety requirements (see section 2.3.2 for custodial facility responsibilities regarding the non-radioactive NDA PDP components).

\subsection{NTP Working Reference Material (WRM) Standards of the NDA PDP}

The following primary isotopes are evaluated under the NDA PDP Plans. Of these, the first four are the most significant in terms of WIPP inventory parameters and limits, and are subject to scoring as specified in this plan.

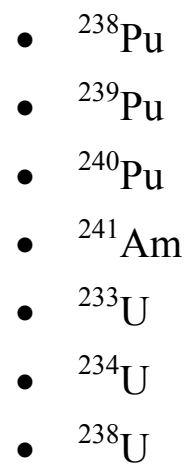

The uranium isotopes comprise a significant fraction of the DOE inventory and must be accounted for in the repository assessments, but are not subject to NDA PDP scoring. Only transuranic alpha emitting isotopes are considered when scoring NDA PDP data. National TRU Program PDP standards containing plutonium (powder and granular forms), americium, and depleted and enriched uranium have been specified, fabricated, and distributed to select sites to support the NDA PDP.

Refer to Appendix E for the general specifications of the NTP WRM standards (also referred to as PDP standards) in the current NTP WRM inventory. Detailed information and data concerning the specification, design, fabrication, and traceability for each standard manufactured in each NTP WRM production phase is provided in its respective production plan document (LAUR-96-2277, LAUR-98-213, LA-CP-00-54, LA-CP-00-110, LA-CP-01-208, LA-CP-030072, LA-CP-03-0763). As indicated in Table 2, seven different PDP standard production campaigns were completed each producing NDA PDP standards with differing nuclear material content. 
Table 2. NDA PDP standard inventory.

\begin{tabular}{cll}
\hline & \multicolumn{1}{c}{ Type of Activity } & Production Phase \\
\hline 1. & Weapons-grade plutonium (low mass set) & Phase I \\
2. & Weapons-grade plutonium (high mass set) & Phase II.A \\
3. & Large particle size weapons-grade plutonium & Phase II.B \\
4. & Enriched uranium & Phase II.C \\
5. & Depleted uranium & Phase II.D \\
6. & Increased Am/Pu ratio (relative to the native quantity in & Phase III.A \\
& weapons-grade plutonium) & \\
7. & Heat source plutonium & Phase III.B \\
\hline
\end{tabular}

\subsection{PDP Surrogate Waste Matrix Drums}

The matrix drums are used with the PDP standards to create test samples containing matrix materials representative of DOE TRU waste types. These matrix materials are contained within the PDP matrix drum in a manner replicating the nominal properties of an actual waste matrix type for material composition, density, and density distribution. In this manner, the drum matrix surrogates replicate the physical properties of real waste forms and manifest perturbations in the response of NDA systems, as do actual wastes.

The NDA PDP matrix drum inventory consists of the following:

- Non-interfering matrix (empty) drum, serial\# 001

- Combustible waste, serial\# 003

- Glass waste, serial\# 004

- Solidified inorganic sludge, serial\# 005

- Mixed metals waste, serial\# 006

The non-interfering matrix drum was established for consideration of NDA response characteristics when virtually no interfering matrix material is present. This "non-interfering" or "empty" matrix is reserved for particular test applications in which the very low density attributes are considered technically appropriate for the evaluation of an NDA system capability. The use of the non-interfering waste matrix is not routinely planned during primary NDA PDP cycles.

Refer to Appendix F for general information on the PDP matrix drum design and configuration. Detailed information regarding the specification, design, and as-built data for the NDA PDP matrix drums is provided in the document INEEL/EXT-02-00320, Design of Interfering Matrix Drums for the Non-Destructive Assay Performance Demonstration Program for the National TRU Program. 
The NDA PDP Coordinator shall coordinate any transfers of NDA PDP drums and components between sites and no such movement will occur without written approval from CBFO. See Appendix B for additional information on shipment and receipt of NDA PDP materials.

\subsection{PROCUREMENT}

Procurement activities necessary for conducting the NDA PDP must comply with the QAPD. In accordance with the QAPD, the responsible purchasing organization maintains all procurement documents and performs all procurement activities.

\subsection{TRAINING}

Each organization involved in the implementation of the NDA PDP shall meet the training requirements of the QAPD. Organizations shall retain on file evidence that: 1) personnel have the necessary program documents (controlled or uncontrolled, as applicable) for their use, and 2) personnel have read and understand program-governing documents pertinent to their duties in support of the NDA PDP. At a minimum, these documents include applicable portions of the QAPD, the WAC, and this plan.

Training for the NDA PDP CFAC and SPT will be conducted for each of the participating facilities prior to their involvement. The training will be conducted using an approved training plan. Successful completion of the training will result in issuance of a certificate of training by the NDA PDP Coordinator that indicates the NDA PDP qualifications of the trained individual. The essential training elements for the CFAC and SPT are as follows:

- Overview of the NDA PDP

- NDA PDP Program Coordination

- NDA PDP Participating Site Responsibilities

- NDA PDP Test Sample Description and Assembly

- NDA PDP Sample Control

- NDA PDP Sample Preparation Responsibilities

- NDA PDP Sample Preparation

- NDA PDP Test Sample Custody

- NDA PDP Test Sample Analysis and Reporting

- NDA PDP Test Sample Return and Disassembly

- NDA PDP Records

Reading material is provided for the initial training through correspondence with the NDA PDP Coordinator. A test is integrated into the training module and must be passed with indicated proficiency before proceeding. Upon successful completion of the initial training, the trainee will participate in hands-on cycle activity training at the respective site. This hands-on training is presented by the custodial facility PDP standards custodian or another individual that has successfully completed the training and has performed the function of an SPT member on a previous cycle. Once the hands-on training is successfully accomplished, the certificate is completed and signed by the NDA PDP Coordinator, the hands-on trainer, and the certification recipient. Copies of the training certificate are distributed as QA records. Once the certificate is 
completed and signed by the NDA PDP Coordinator, the individual is qualified to conduct the NDA Drum PDP activities indicated on the certificate.

\subsection{QA RECORDS}

Records generated by the NDA PDP and participating sites during the conduct of a PDP cycle are QA records. All NDA PDP cycle documentation must be maintained in a traceable and auditable condition. Storage conditions and duration must meet the requirements of the QAPD and other implementing QA documents and procedures.

The minimum QA records for the NDA PDP are identified and listed below in accordance with the QAPD requirements. In addition, CBFO and/or the NDA PDP Coordinator may determine that records of other program activities are QA records and enter them into the QA records system with the same level of control and maintenance.

These QA records may be organized by NDA PDP Plan revision, by NDA PDP cycle, or by other principle, as applicable. These records are nonpermanent records and shall be maintained in accordance with the QAPD requirements. Records disposition, when applicable, will be in accordance with $\mathrm{CBFO} / \mathrm{NTP}$ requirements, approved procedures, and work plans.

All QA records identified in this plan shall be stored in accordance with records storage requirements in the QAPD. Access to QA records will be limited to personnel involved in the program or having related QA or records custodial responsibilities.

The following documents will be maintained as QA records for the NDA PDP:

- PDP plans (all revisions)

- Procurement records

- Radioactive standard and matrix drum design and production records (each drum and PDP standard production phase)

- $\quad$ SPT training (training materials, test records, certificates)

- Assay System Registration Forms

- Records of cycle set-up (notification letters, shipping records, and other correspondence)

- Participant assay reports and supporting forms (assay data report forms, chain-ofcustody records, and configuration forms)

- Scoring reports

- Reviews of corrective actions and supporting data and recommendations made to $\mathrm{CBFO}$

The following matrix of QA records provides a more detailed listing of records and designates responsibility for maintenance. 
NDA PDP Documentation/Records List

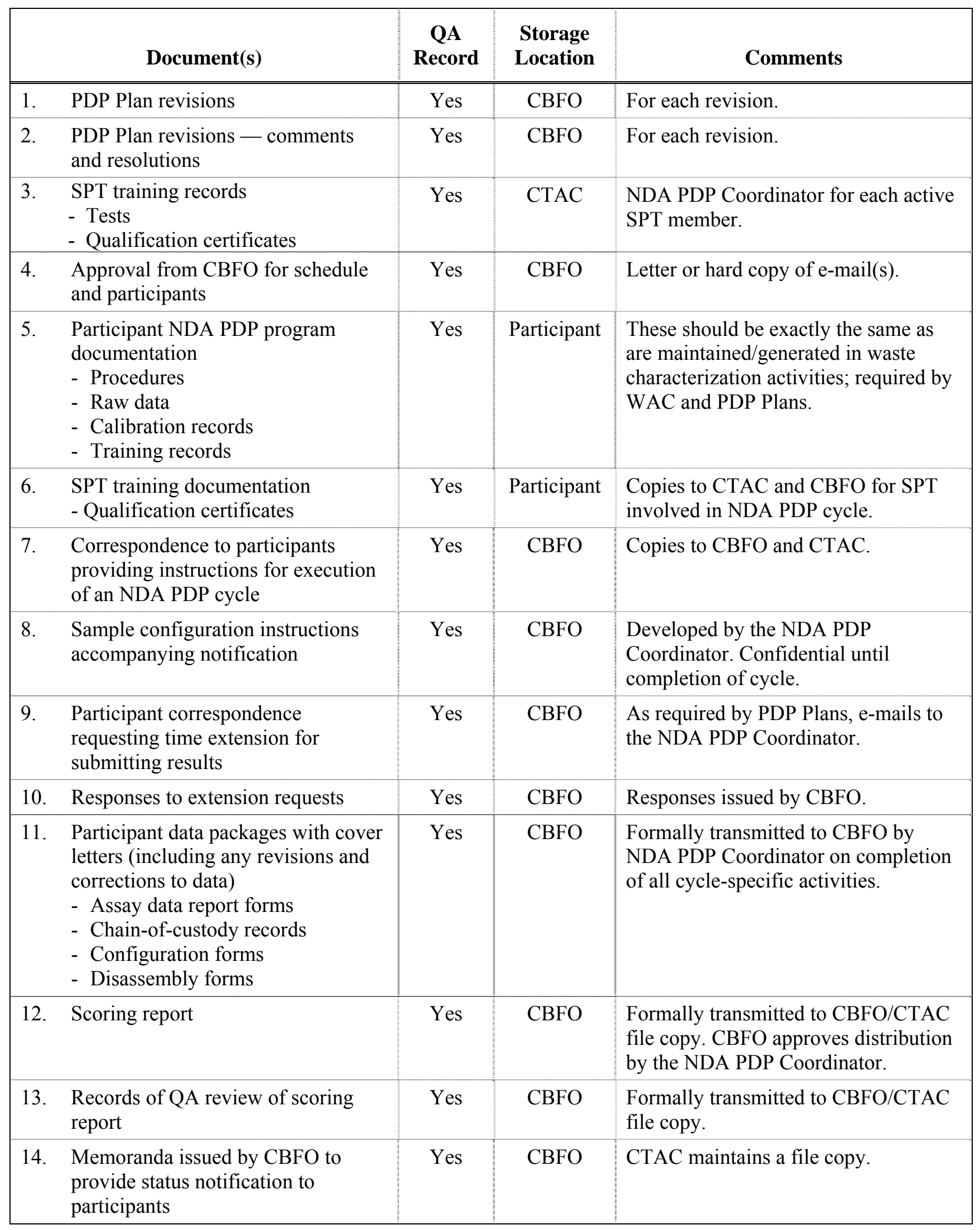




\begin{tabular}{|c|c|c|c|}
\hline Document(s) & $\begin{array}{c}\text { QA } \\
\text { Record }\end{array}$ & $\begin{array}{l}\text { Storage } \\
\text { Location }\end{array}$ & Comments \\
\hline $\begin{array}{l}\text { 15. Transmittal letter with cycle-specific } \\
\text { records }\end{array}$ & Yes & $\mathrm{CBFO}$ & $\begin{array}{l}\text { Transmitted upon completion and } \\
\text { receipt of all cycle records. }\end{array}$ \\
\hline $\begin{array}{l}\text { 16. Current document, software revision } \\
\text { list, and software verification and } \\
\text { validation documentation }\end{array}$ & Yes & $\mathrm{CBFO}$ & Required by the CBFO QAPD. \\
\hline 17. NDA PDP MP & Yes & CTAC & $\begin{array}{l}\mathrm{CBFO} \text { reviews to ensure MP meets } \\
\text { CBFO requirements. }\end{array}$ \\
\hline $\begin{array}{l}\text { 18. Participants' reports of corrective } \\
\text { actions }\end{array}$ & Yes & $\mathrm{CBFO}$ & Submitted to CBFO. \\
\hline
\end{tabular}




\subsection{GLOSSARY}

ASSAY COORDINATOR - There are two types of assay coordinators functioning in the NDA PDP. One, referred to as the Custodial Facility Assay Coordinator (CFAC) is the facility point-of-contact responsible for receipt of the cycle notification letter, proper sample preparation team (SPT) documentation, and return of the SPT documentation to the NDA PDP Coordinator in a timely manner. A CFAC can be an active participant in the NDA PDP cycle as an SPT member.

The second type of assay coordinator is the Measurement Organization Assay Coordinator (MOAC). This person is generally responsible as the point-of-contact for cycle measurement results, corrective action process, review of draft approval status notification memoranda, and other activities related to the measurement operations of the NDA PDP cycle. It is possible that the CFAC and MOAC roles can be filled by the same individual except that the MOAC cannot participate as an active member of the SPT.

BIAS - The systematic error component of the total uncertainty, that is, a constant positive or negative deviation of the method average from the correct value or an accepted reference value under specific measurement conditions. The percent recovery $(\% \mathrm{R})$ in the NDA PDP summary report includes both systematic (bias) and non-systematic (precision) uncertainty.

CORRECTIVE ACTION - Measures taken to rectify conditions adverse to quality and, where necessary, to preclude their recurrence.

CUSTODIAL FACILITY - The physical and organizational entity responsible for the storage, care, and proper handling of NDA PDP materials during cycle activities and when not in use for NDA PDP activities.

INACTIVE SYSTEM - A CBFO-approved and operational NDA system not being used to characterize WIPP wastes for the NTP at the time a primary NDA PDP cycle is conducted.

MATRIX DRUM - 208-liter (55-gallon) steel drum designed, fabricated and serial-numbered for the PDP, containing a surrogate waste matrix configuration representative of a specified population of the actual DOE waste inventory. The interfering or non-interfering matrix drum contains an internal structure for insertion and positioning of PDP standards and a 90 mil polyethylene liner.

MEASUREMENT ORGANIZATION - The entity responsible for the assay of NDA PDP samples and WIPP waste. Frequently this entity is not the same company or entity that functions as the custodial facility or site facility contractor.

MEASUREMENT SYSTEM - The individual NDA instrument that has received a unique NDA PDP registration number. A measurement system may function with various independent modes of assay.

NDA PDP COORDINATOR - An individual responsible for coordinating the technical operations of the NDA PDP, including designation of NDA PDP cycle-specific sample component configuration, procurement of materials and services required for replacement and/or manufacture of new NDA PDP sample components (i.e., NDA PDP matrix drums, and consumables), SPT oversight, scheduling NDA PDP cycles, scoring NDA PDP data received from participants, summary report generation, and preparation of other NDA PDP-related CBFO documents as directed and for approval by the CBFO PDP Appointee. 
NDA PDP SAMPLE - A blind sample prepared and sealed by the SPT for subsequent analysis by a measurement system for qualification under the PDP. A PDP sample for the NDA Drum PDP is comprised of a 55-gallon matrix drum and PDP standards installed in accordance with instructions received from the NDA PDP coordinator. Sample matrix and source characteristics will representatively span nominal WIPP waste characteristics expected to be received for characterization by the measurement organization including, but not limited to, isotopics, plutonium concentration, $(\alpha, n)$ reactions, interfering matrices, and source distribution.

NDA PDP STANDARD - A radioactive source specifically designed, prepared, or acquired and certified for the NDA PDP.

NONDESTRUCTIVE ASSAY - Assay methods for waste items that do not affect the physical or chemical form of the material.

NON-INTERFERING MATRIX - Specifies a drum sample that contains only the supports for the PDP standards (i.e., no installed matrix material), and serves as the non-interfering matrix test sample.

PDP MANAGER - An individual within the Program Coordinator organization responsible for overall performance of the NDA PDP and other elements of the PDP (Headspace Gas and RCRA Constituent Analysis of Solidified Wastes).

PROGRAM COORDINATOR - A CBFO-designated organization that administers and coordinates PDP functions. The program coordinator will designate the PDP Manager.

PDP STANDARDS CONFIGURATION ATTESTANT - A member of the two-person SPT responsible for verifying the proper configuration of the NDA PDP sample, including emplacement of NDA PDP standards and performing sample security-related procedures.

PDP STANDARDS CUSTODIAN - The lead member of the SPT responsible for coordination of on-site NDA PDP sample preparation activities.

PRECISION - A measure of the variance among individual measurements of the same property made under prescribed conditions. Precision is represented in NDA PDP summary reports as a percent relative standard deviation (\%RSD).

PRIMARY CYCLE - The annual NDA PDP cycle. The primary cycle is included in the Program Coordinator's master project schedules for the NDA PDP and occurs at approximately the same time each year to ensure a site receives the opportunity to gain NDA PDP approval every 12 months.

PROGRAM COORDINATOR - A CBFO-designated organization that administers and coordinates PDP functions. The program coordinator will designate the PDP manager.

SAMPLE PREPARATION PROCEDURE - A procedure generated by the NDA PDP Coordinator for each facility measurement organization for each NDA PDP cycle. This procedure provides instructions to the SPT on configuration of the NDA PDP sample (i.e., emplacement of NDA PDP standards within a specified configuration NDA PDP drum).

SAMPLE PREPARATION TEAM (SPT) - A two-person team, consisting of an NDA PDP standards custodian and PDP standards configuration attestant that prepare and certify NDA PDP samples for a given facility measurement organization. The SPT is responsible for ensuring that each NDA PDP sample is prepared according to the NDA PDP sample preparation procedures provided by the NDA PDP Coordinator. In addition, the SPT ensures proper disassembly and 
return to storage of all NDA PDP components after analysis by the facility measurement organization.

SUPPLEMENTAL CYCLE - An NDA PDP cycle in addition to the annual primary NDA PDP cycle. Reasons that a supplemental cycle may be necessary include accommodation of measurement facilities unable to participate in the primary cycle, to support implementation of new or modified systems, or to perform specific testing as directed by the CBFO. Additional supplemental cycles may be conducted on an as-needed basis at CBFO direction.

TOTAL MEAUSUREMENT UNCERTAINTY - The propagated measurement error potential from all bias and precision sources including interference effects such as variable matrices, isotopic compositions, spatial distributions, contaminating radionuclides, and others. 


\subsection{REFERENCES}

CBFO-94-1012, Quality Assurance Program Document, current revision, U.S. Department of Energy Carlsbad Field Office, Carlsbad, New Mexico.

DOE/CAO-95-1121, Transuranic Waste Baseline Inventory Report, current revision, U.S. Department of Energy Carlsbad Field Office, Carlsbad, New Mexico.

DOE/WIPP-02-3122, Transuranic Waste Acceptance Criteria for the Waste Isolation Pilot Plant, current revision, Waste Isolation Pilot Plant, U.S. Department of Energy, Carlsbad, New Mexico.

DOE/CBFO-01-1006, Performance Demonstration Program Plan for Nondestructive Assay of Boxed Wastes for the TRU Waste Characterization Program, current revision, U.S. Department of Energy Carlsbad Field Office, Carlsbad, New Mexico.

DOE/CBFO-01-3107, Performance Demonstration Program Management Plan, current revision, U.S. Department of Energy Carlsbad Field Office, Carlsbad, New Mexico.

INEL-94/0104, Performance Demonstration Program for Nondestructive Assay for the TRU Waste Characterization Program, Initial Cycle Source Design, Idaho National Engineering and Environmental Laboratory, Lockheed Martin Idaho Technologies Company, LLC

INEEL/EXT-02-00320, Design of Interfering Matrix Drums for the Non-Destructive Waste Assay Performance Demonstration Program for the National TRU Program, February 2002, Idaho National Engineering and Environmental Laboratory, Bechtel BWXT Idaho, LLC, Idaho Falls, Idaho.

LA-CP-00-54, National Transuranic Waste Program Nondestructive Assay Performance Demonstration Program Enriched Uranium Production Plan - Phase II.C, current revision, Los Alamos National Laboratory, Los Alamos, New Mexico.

LA-CP-00-110, National TRU Waste Program NDA PDP Depleted Uranium Production Plan - Phase II.D, current revision, Los Alamos National Laboratory, Los Alamos, New Mexico.

LA-CP-01-208, National TRU Waste Program Non-Destructive Assay Performance Demonstration Program Large Particle Working Reference Material Production Plan - Phase II.B, current revision, Los Alamos National Laboratory, Los Alamos, New Mexico.

LA-CP-03-0072, National TRU Waste Program Non-Destructive Assay Performance Demonstration Program Increased Americium: Plutonium Working Reference Material Production Plan - Phase III.A, current revision, Los Alamos National Laboratory, Los Alamos, New Mexico.

LA-CP-03-0763, National TRU Waste Program Non-Destructive Assay Performance Demonstration Program Heat Source Plutonium Working Reference Material Production Plan - Phase III.B, current revision, Los Alamos National Laboratory, Los Alamos, New Mexico.

LAUR-96-2277, NDA PDP Working Reference Material Productions Plan - Phase I, current revision, Los Alamos National Laboratory, Los Alamos, New Mexico.

LAUR-98-213, CST-8-PLA-STD-106, NDA PDP High Mass Working Reference Material Production Plan - Phase II.A, current revision, Los Alamos National Laboratory, Los Alamos, New Mexico. 


\section{Appendix A}

\section{NDA Performance Demonstration Program Forms}




\section{Appendix A NDA PDP Forms}

This appendix contains forms that are essential to the conduct and QA record of the NDA Drum PDP cycles. There are four forms included - the purpose of each is described below. Each of the forms is called out in the body of this plan, under the Responsibilities section, 2.0.

1. The Nondestructive Assay Drum PDP Sample Configuration Form is the primary instruction document provided to the SPT by the NDA PDP Coordinator for the purpose of assembly and documented disassembly of a cycle test sample. This form is initiated by the NDA PDP Coordinator for instruction purposes but becomes one of the primary QA record documents as it is completed by the SPT during stages of the NDA Drum PDP cycle. The documentation on this form provides essential evidence that the proper NDA PDP standards were placed in the positions as originally designated on the form. Any discrepancies from the original instruction discovered during disassembly must be documented for quality assurance purposes. The completed form is a QA record that must be returned to the NDA PDP Coordinator upon disassembly of the sample as soon after the sample is returned to the SPT as is reasonable.

2. The Nondestructive Assay Drum PDP Sample Custody Form is utilized by the SPT and the measurement organization to indicate the time during which each entity is in control of the sample. This document is initiated by the SPT and completed by the SPT upon disassembly. The measurement organization assay coordinator (MOAC) or designee is responsible for the integrity of the NDA PDP samples during the time that the sample is in their possession. The custody form is to be signed and dated by the MOAC or designee for that period of sample possession. The completed form is a QA record that must be returned to the NDA PDP Coordinator upon disassembly of the sample as soon after the sample is returned to the SPT as is reasonable.

3. The Nondestructive Assay PDP Report Form contains the headings and spaces for the essential information required by the NDA PDP for each measurement of a test sample. This form must be utilized by the MOAC or designee to present the information to the NDA PDP Coordinator once all data review by the measurement organization is complete. One Nondestructive Assay PDP Report Form is required for each NDA Drum PDP sample analyzed and for each NDA system used to analyze the sample. The completed forms, signed and dated, are QA records of the NDA PDP cycle.

4. The NDA PDP Participating Facility Contacts Form is provided for convenience, to be utilized by the custodial facility assay coordinator (CFAC) or supervisor thereof, to supply the NDA PDP Coordinator with the names and contact information of those individuals who have responsibility for the conduct and management of the NDA PDP at the WIPP waste packaging and measurement facility. The Responsibilities section, 2.0, of this document provides the activity expectations of the personnel assigned to the NDA PDP functional titles. In some cases, one individual could function in more than one titled capacity. Though this contact information is essential to the conduct of the NDA PDP, the completed document is not an NDA PDP QA record. 


\section{Nondestructive Assay Drum PDP Sample Configuration Form}

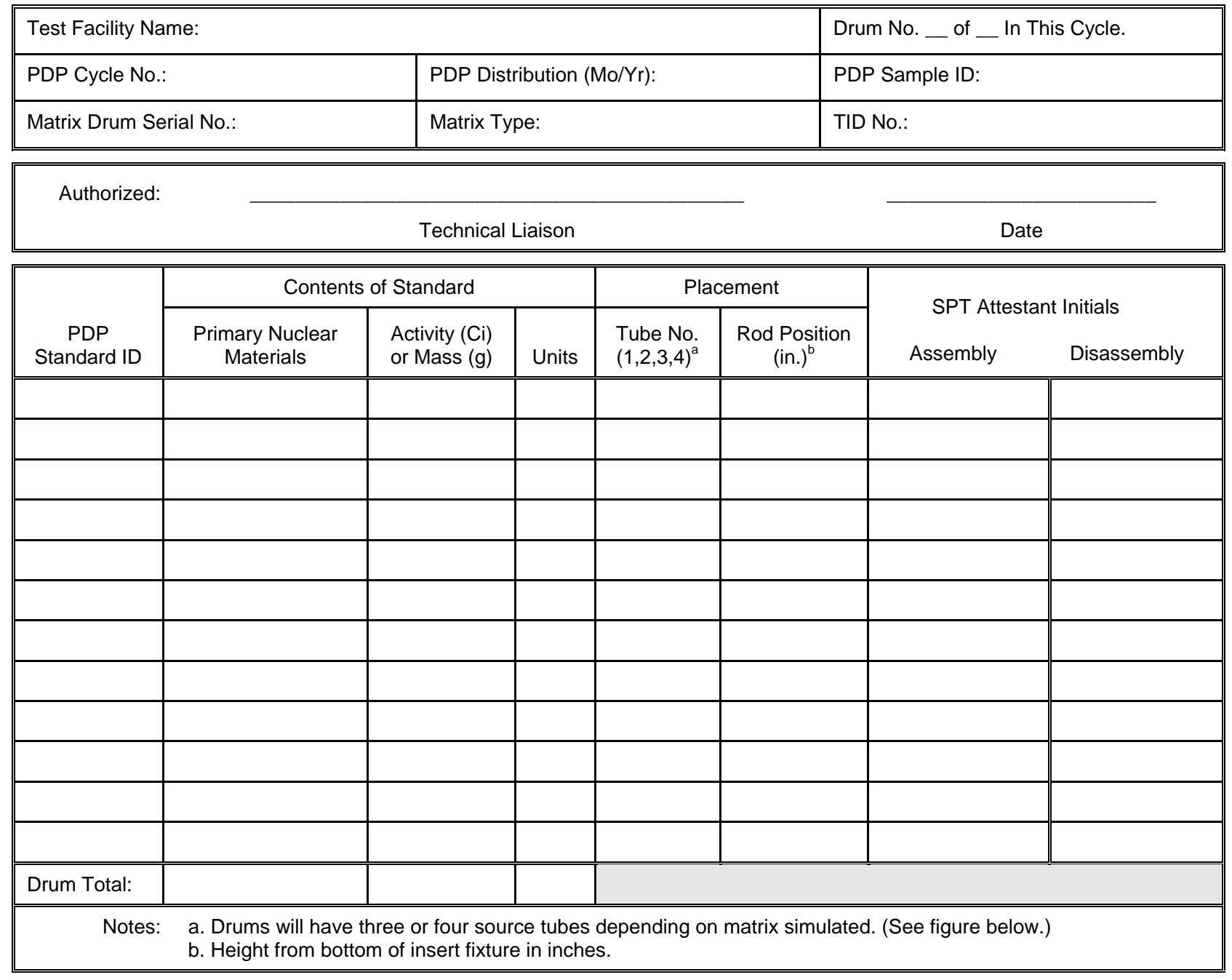

Signatures:

PDP Standards Custodian

Date
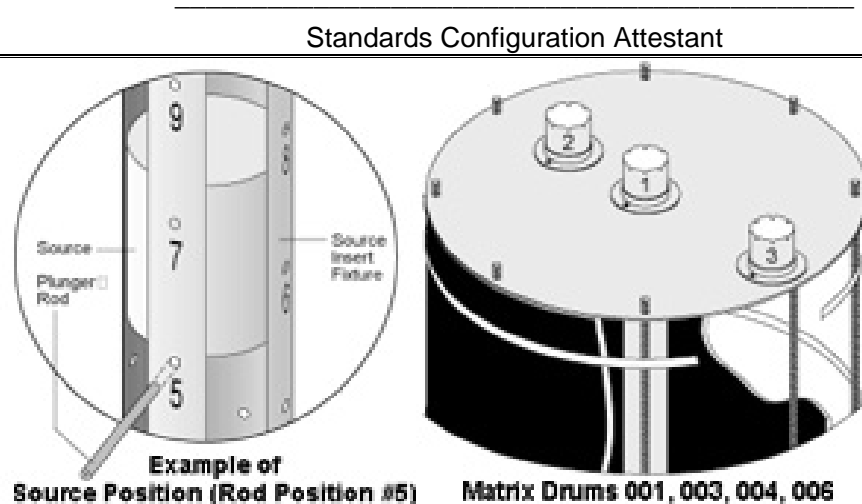

Matrix Dnums 001, 003, 004, 006

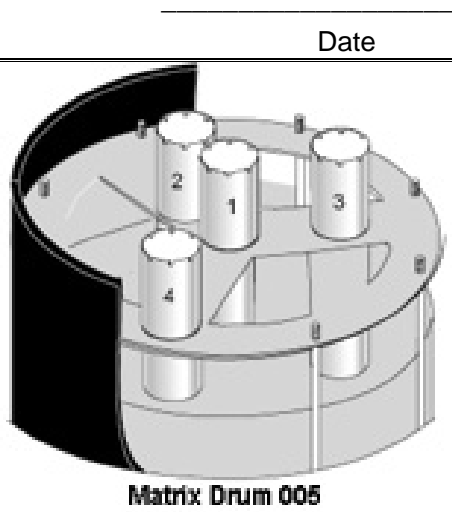




\section{Nondestructive Assay Drum PDP Sample Custody Form}

Drum Serial Number:

Assay Site:

TID Serial Number:

Distribution Cycle Number:

Comments:

\section{Sample Preparation}

Sample Preparation Date:

PDP Standards Custodian

Date

Initials

Standards properly placed:

Matrix drum TID properly sealed:

Sample information form attached and sealed:

Standards Configuration Attestant

Date

\begin{tabular}{||l|l|l|l||}
\hline \hline Relinquished by: & Date/Time & Received by: & Date/Time \\
\hline & & & \\
\hline \\
\hline
\end{tabular}

White: NDA PDP coordinator copy on final disposition

Canary: SPT copy on final disposition

Pink: NDA PDP coordinator copy

Gold: SPT copy 


\section{Nondestructive Assay Performance Demonstration Program Report Form}

Laboratory ID: PDP Cycle: Drum Serial No.:
Assay Facility:

Supplemental Cycle: Laboratory Sample ID:

Replicate:

of

Final Result Summary

\begin{tabular}{||l|l|l||}
\hline \multicolumn{1}{|c|}{ Parameter } & Final Result & Total Uncertainty (One Standard Deviation) \\
\hline Total ${ }^{239} \mathrm{Pu}$ fissile gram equivalent $(\mathrm{g})$ & & \\
\hline Total TRU alpha activity (curies) & & \\
\hline Thermal Power (W) & & \\
\hline
\end{tabular}

Method Summary

\begin{tabular}{|c|c|c|c|c|c|c|}
\hline & \multirow[b]{2}{*}{ Identification } & \multirow[b]{2}{*}{ Classification } & \multirow{2}{*}{$\begin{array}{l}\text { Associated SOP Identification } \\
\text { (Including Revision No.) }\end{array}$} & \multirow{2}{*}{$\begin{array}{l}\text { Count Time } \\
(\min )\end{array}$} & \multicolumn{2}{|c|}{ Analysis } \\
\hline & & & & & Date & Time \\
\hline Method 1 & & & & & & \\
\hline Method 2 & & & & & & \\
\hline Method 3 & & & & & & \\
\hline
\end{tabular}

Individual Isotope Data

\begin{tabular}{|c|c|c|c|c|c|c|c|c|}
\hline \multirow[b]{2}{*}{ Isotope } & \multirow[b]{2}{*}{ Activity Result } & \multicolumn{2}{|c|}{ Uncertainty (1sd) } & \multicolumn{4}{|c|}{ Quantification Method } & \multirow{2}{*}{$\begin{array}{c}\text { Method } \\
\text { Number } \\
\text { (From } \\
\text { Summary) }\end{array}$} \\
\hline & & Count & Total & Direct & Ratio & $\begin{array}{l}\text { Scaling } \\
\text { Isotope }\end{array}$ & $\begin{array}{l}\text { Ratio } \\
\text { Value }\end{array}$ & \\
\hline \multicolumn{9}{|l|}{${ }^{238} \mathrm{Pu}$} \\
\hline \multicolumn{9}{|l|}{${ }^{239} \mathrm{Pu}$} \\
\hline \multicolumn{9}{|l|}{${ }^{240} \mathrm{Pu}$} \\
\hline \multicolumn{9}{|l|}{${ }^{241} \mathrm{Am}$} \\
\hline & & & & & & & & \\
\hline & & & & & & & & \\
\hline & & & & & & & & \\
\hline & & & & & & & & \\
\hline
\end{tabular}

Comments:

Approval: 
NDA PDP Participating Facility Contacts

\begin{tabular}{|c|c|c|c|}
\hline NAME & MAIL ADDRESS & $\begin{array}{l}\text { PHONE/FAX/E- } \\
\text { MAIL }\end{array}$ & NOTE \\
\hline \multicolumn{4}{|c|}{ Custodial Facility Management Contacts } \\
\hline Operations & & $\begin{array}{l}\text { Voice: } \\
\text { Fax: } \\
\text { E-mail: } \\
\text { Cell: }\end{array}$ & \\
\hline SNM Custodian & & $\begin{array}{l}\text { Cell Voice: } \\
\text { Fax: } \\
\text { E-mail: } \\
\text { Cell: }\end{array}$ & \\
\hline \multicolumn{4}{|c|}{ Custodial Facility NDA PDP Function Contacts } \\
\hline \multicolumn{4}{|c|}{ Assay Coordinator } \\
\hline & & $\begin{array}{l}\text { Cell Voice: } \\
\text { Fax: } \\
\text { E-mail: } \\
\text { Cell: }\end{array}$ & \\
\hline \multicolumn{4}{|c|}{ Components Custodian } \\
\hline & & $\begin{array}{l}\text { Cell Voice: } \\
\text { Fax: } \\
\text { E-mail: } \\
\text { Cell: }\end{array}$ & \\
\hline \multicolumn{4}{|c|}{ PDP Standards Custodian } \\
\hline & & $\begin{array}{l}\text { Cell Voice: } \\
\text { Fax: } \\
\text { E-mail: } \\
\text { Cell: }\end{array}$ & \\
\hline \multicolumn{4}{|c|}{ PDP Standards Configuration Attestant } \\
\hline & & $\begin{array}{l}\text { Cell Voice: } \\
\text { Fax: } \\
\text { E-mail: } \\
\text { Cell: }\end{array}$ & \\
\hline
\end{tabular}




\section{Appendix B}

\section{Shipment and Receipt of NDA PDP Materials}




\section{Appendix B Shipment and Receipt of NDA PDP Materials}

This appendix describes the policies and some processes of shipping and receiving of NDA PDP materials. This appendix is not meant to be all inclusive of the requirements that will be encountered for movement of NDA PDP materials. In addition to these policies and process guidelines, the shipment and receipt of all NDA PDP materials must meet federal, state, and facility requirements for both the shipping and receiving facility.

\section{B.1 GENERAL}

The NDA PDP Coordinator shall coordinate any transfers of NDA PDP materials between sites and no transfers will occur without written approval from CBFO. The NDA PDP Coordinator will provide the transferring facility with the necessary contact information (names, phone numbers, e-mails, and physical addresses) for the receiving facility. The current custodial facility will notify each receiving site contact prior to the shipping date for NDA PDP materials. The materials will be sent to the address and individual designated by the receiving facility.

The custodial facility assay coordinator at each facility shall notify the NDA PDP Coordinator in writing (e-mail is acceptable), of any changes in contact personnel.

\section{B.2 PACKAGING OF NDA PDP MATRIX DRUMS FOR FREIGHT SHIPPING}

Transport conditions entail vibration, heavy equipment handling as well as potential exposure to moisture. Such conditions require that the PDP matrix drum be properly packaged to prevent internal alteration of the matrix media and/or external damage. Minimal requirements for packaging an NDA PDP matrix drum for shipment are listed below.

1) Transport of the NDA PDP matrix drum requires that it be strapped to a pallet of sufficiently robust construction to accommodate the weight of the drum. One drum per pallet.

2) For transport, a rubber pad, thick cardboard or equivalent material, serving to dampen vibration should be placed between the drum bottom and the upper surface of the pallet.

3) To prepare the drum for transport, place all matrix spacers within the aluminum insert fixtures and insert each fixture into its respective drum insert tube. Source/spacer locating pins are also to be installed in the insert fixture locating holes. For those cases where there are not enough insert fixture holes to install all of the locating pins, place the pins into a plastic bag and tape to the top of the drum. Finally thread the wire security lanyard through the three (or four in the case of sludge) rings of the insert fixture top caps and fix the loop end to the lock chime bolt.

4) To prevent moisture from entering the drum during transport drum covers have been provided. To further protect against damage to the labeling and exterior finish, the complete exterior of the drum must be wrapped in bubble wrap or cardboard followed by a thorough application of stretch-wrap plastic. The stretch-wrap must cover all sides and in particular secure the top such that no water can enter the drum interior.

5) The drum is to be secured to the pallet by positioning the drum such that the three insert fixture penetrations align parallel with the pallet wood plates. Place a piece of $2 \times 4$ wood approximately 28 inches in length, centered on the top lid and aligned along the side of the three insert fixture top caps. The wood oriented in this way provides protection for the insert fixtures and tubes and provides a mount point to attach steel strapping.

6) Steel strapping is required to fasten the drum to the pallet, $3 / 4$ inch wide steel strapping is to be used. The strapping is to run under the top pallet wood plates and up around the $2 \times 4$, tightened and retained in place. This same operation is to be performed on the opposite end of the $2 \times 4$. The second strap is then ratcheted down to firmly hold the drum to the pallet. Use two clips or heavy staples to secure each strap in place. (For the case of the inorganic sludge drum which has 
four insert fixtures, position the $2 \times 4$ wood piece parallel to the wood plates of the pallet but ensure the $2 \times 4$ does not impinge upon any of the insert fixture caps.)

7) In addition to the shipping label, ensure that a minimum of two signs stating DO NOT STACK are conspicuously posted on the outer surface of the stretch-wrap plastic.

\section{B.3 RECEIPT OF NDA PDP MATRIX DRUMS AND COMPONENTS}

On receipt of NDA PDP matrix drums and components, the PDP sample components custodian shall:

- verify that the serial numbers and physical descriptions of the NDA PDP matrix drums and associated components received match those listed on the shipping manifest.

- ensure the matrix spacers are included in the shipment as indicated on the manifest. The PDP matrix drum insert fixtures will need to be removed to assess the presence of the spacers.

- open any additional shipped packages to verify the content and quantity of items.

- verify that the matrix drums and ancillary components have not been damaged during shipping. Where shipping manifest discrepancies are noted or damage is found, the assay coordinator and/or the SPT shall secure the items and the assay coordinator and/or the SPT shall notify the NDA PDP Coordinator. If no discrepancy or damage is found, the assay coordinator shall notify the NDA PDP Coordinator in writing or by e-mail, that the shipment was received in acceptable condition.

\section{B.4 TRANSFER OF NTP WRM STANDARDS (PDP STANDARDS)}

All transfers of NTP WRM standards must have prior written approval from CBFO.

Before shipment of PDP standards, each receiving custodial facility will make appropriate arrangements with the facility's safeguards and radiation safety organizations for storage and accountability. Generally, the designated PDP standards custodian will coordinate with the site safeguards staff to comply with all site special nuclear material (SNM) requirements. All shipping details and arrangements will occur between the trained staff of the DOE facilities of both the shipping and receiving entities. The NDA PDP Coordinator will assist in facilitating the transfer and should remain informed by the shipping and receiving facilities of communications and significant activities associated with the transfer.

At the time of receipt, the PDP custodial facility assay coordinator and/or PDP standards custodian assigned by the custodial facility shall inspect, inventory, and secure the PDP standards. Documentation of these activities must then be provided to the NDA PDP Coordinator. 


\section{Appendix C}

\section{NDA Drum PDP Registration Form}




\section{Appendix C \\ NDA Drum PDP System Registration Form}

This appendix contains the Registration Form that must be completed by the MOAC or designee, and revised when the information is no longer correct, for an NDA system to be authorized by CBFO to participate in the NDA Drum PDP.

\section{General Instructions}

1. Registration Forms are to be completed and returned to the NDA PDP Coordinator at least three (3) weeks prior to participation in the PDP.

2. Separate registration is required for each NDA measurement system.

3. After initial submission, the forms need be resubmitted only when a change is made in the registration information.

4. The NDA PDP Coordinator will acknowledge all registration requests and assign a tracking identifier to each registered system.

5. The NDA PDP Coordinator will maintain a current list of all registered systems.

\section{Instructions for Specific Questions}

\section{$\underline{\text { Section A }}$}

1. Enter the full formal name of the measurement system.

2. Enter the acronym by which the system should be referenced.

3. Enter a number associated with this unit (if applicable).

4. Check the appropriate descriptor. "Fixed, Permanent" indicates that the system was intended to be installed permanently at the current location. "Transportable, Nonpermanent" indicates a long-term installation that can be relocated. "Mobile, Trailer" indicates systems intended for routine movement between sites for short-term contracts.

5. Enter the DOE site where the system will be installed for NDA PDP cycle participation.

6. Enter the on-site location designator for the system.

7. Enter the name of the institution/facility/company that owns the system.

8. Enter the name of the institution/facility/company that operates the system.

9. Enter the name of the person who should be contacted for information on the system.

10. Enter the title of the person identified in box 9 .

11. Enter the affiliation of the person identified in box 9 .

12. Enter the mailing address for the person identified in box 9 .

13. Enter the express package delivery address (i.e., street address, not a P.O. address) for the person identified in box 9.

14. Enter the e-mail address for the person identified in box 9.

15. Enter the phone number for the person identified in box 9 .

16. Enter the fax number for the person identified in box 9 . 


\section{$\underline{\text { Section B }}$}

1. Enter a description of the system, its principles of operation, and optional modes for assay.

2. Enter the identifier(s) for the written standard operating procedures (SOPs) that are used to operate the system for waste assay.

3. For each measurement mode of the system that may be used, enter the measurement principle (gamma, neutron), mode identification (active neutron, etc.), the procedure documenting selection criteria for selecting the subject modes, and the source of isotopic data used for that mode. Sources of isotopic data may be coded:

$\mathrm{P} \quad=\quad$ isotopic data are measured as an integral part of the primary quantitative assay (e.g., gamma spectrometric methods used for both the quantitative and isotopic data).

$\mathrm{S}=\quad$ isotopic data are derived from a secondary method (e.g., the primary quantitation is by passive neutron assay, but isotopic ratios are obtained from an independent gamma spectrometric method).

$\mathrm{AK}=$ isotopic data are obtained from acceptable knowledge of the waste stream or container.

\section{$\underline{\text { Section C }}$}

1 through 5. Check off Yes or No to each question. For each "Yes," indicate the applicable mode(s) from section B.3.

6. Enter the possible mode(s) from section B.3 for each combination of activity range and waste type. Enter "NA" for combinations for which the system will not be used. If explanatory information is required, enter a number in the comments column and add the number and explanation to section D. For example, a system may have a calibration cut-off that falls at the midpoint of a test range. This may be indicated by accepting the range, but specifying a numerical limit in the comment.

7 through 9. Enter the values and units for any limits on NDA PDP tests that, if exceeded, would prevent the system from assaying an NDA PDP sample.

\section{$\underline{\text { Section D }}$}

Add any comments necessary to explain answers in any prior sections or supplemental information useful to the NDA PDP Coordinator in planning effective NDA PDP tests for the system. Attach continuation sheets as needed.

\section{$\underline{\text { Section E }}$}

Enter the requested information for the person submitting the registration form. Sign and forward the original of the form to the NDA PDP Coordinator. 


\section{NDA Drum PDP System Registration Form}

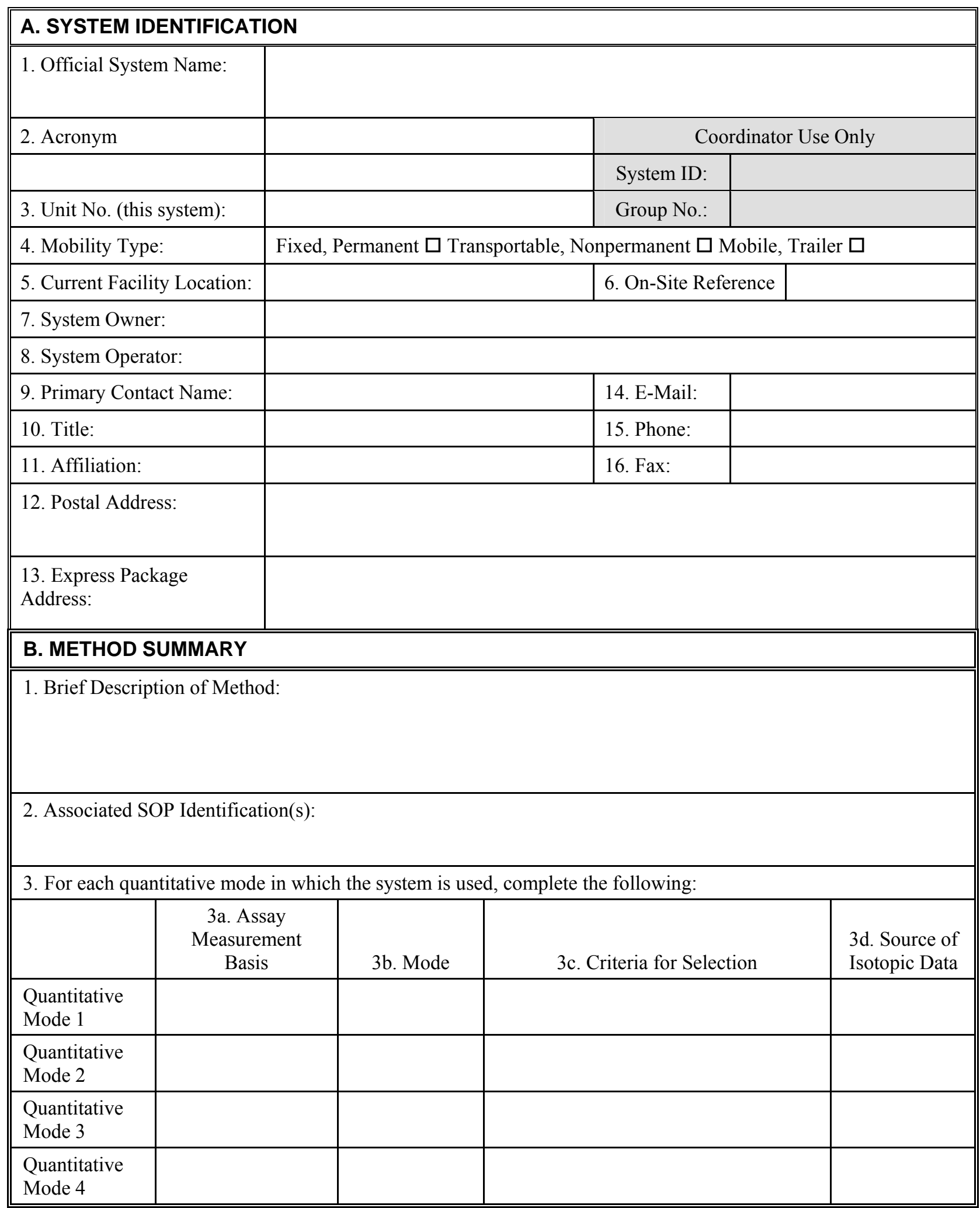




\section{SCOPE AND LIMITATIONS ON SYSTEM USE}

\begin{tabular}{|c|c|c|c|c|c|c|c|}
\hline & & & & & YES & $\mathrm{NO}$ & If YES, Mode No(s): \\
\hline $\begin{array}{l}\text { 1. Will th } \\
\text { contai }\end{array}$ & $\begin{array}{l}\text { ystem be used } \\
\mathrm{g} \text { less than } 0.01\end{array}$ & $\begin{array}{l}\text { certify waste a } \\
\text { uries of TRU is }\end{array}$ & $\begin{array}{l}\mathrm{J}, \text { for } \mathrm{dr} \\
\mathrm{s} ?\end{array}$ & & & & \\
\hline $\begin{array}{l}\text { 2. Will th } \\
\text { pluton }\end{array}$ & $\begin{array}{l}\text { ystem be used } \\
\text { n? }\end{array}$ & $r$ wastes contai & veapons & & & & \\
\hline $\begin{array}{l}\text { 3. Will th } \\
\text { pluton }\end{array}$ & $\begin{array}{l}\text { ystem be used } \\
\text { n? }\end{array}$ & r wastes contaiı & eat sour & & & & \\
\hline $\begin{array}{l}\text { 4. Will tl } \\
\text { ratios? }\end{array}$ & ystem be used & $r$ wastes contaiı & nknowr & & & & \\
\hline 5. Will th & ystem be used & r wastes contail & ranium & & & & \\
\hline $\begin{array}{l}\text { 6. Indica } \\
\text { intend } \\
\text { applic }\end{array}$ & $\begin{array}{l}\text { he ranges and } y \\
\text { articipation in } \mathrm{t} \\
\text { e mode(s) for } \mathrm{e}\end{array}$ & $\begin{array}{l}\text { ste types (with } \\
\text { NDA PDP. Fo } \\
\text { h case. }\end{array}$ & $\begin{array}{l}\text { nucle: } \\
\text { ns wit }\end{array}$ & $\begin{array}{l}\text { Iction char } \\
\text { le quantit }\end{array}$ & $\begin{array}{l}\text { racte } \\
\text { tativ }\end{array}$ & $\begin{array}{l}\text { es) for } \\
\text { des, b }\end{array}$ & $\begin{array}{l}\text { which the system } \\
\text { sure to indicate the }\end{array}$ \\
\hline $\begin{array}{l}\text { Activity } \\
\text { range }\end{array}$ & $\begin{array}{c}\text { Range in } \\
\text { alpha-curies }\end{array}$ & Combustibles & Glass & $\begin{array}{c}\text { Inorganic } \\
\text { Sludge }\end{array}$ & & Metals & Comment No. \\
\hline Low & $>0$ to 0.02 & & & & & & \\
\hline Mid-Low & $>0.02$ to 0.2 & & & & & & \\
\hline Mid-High & $>0.2$ to 2.0 & & & & & & \\
\hline High & $>2.0$ & & & & & & \\
\hline
\end{tabular}

a. Range of TRU activity in a drum; units are curies of alpha-emitting TRU isotopes with half-lives greater than 20 years.

7. Indicate the maximum activity permissible (including units) for testing the system due to calibration, radiological safety, or administrative limits:

8. Indicate the maximum weight permissible (including units) for testing the system due to calibration, safety, or administrative limits:

9. Indicate any other limitations on system tests: (Explanation in Comment No. .)

\begin{tabular}{|l|l|}
\hline Value: & Units: \\
\hline Value: & Units: \\
\hline Value: & Units: \\
\hline
\end{tabular}

\section{COMMENTS (Please add any information relative to participation of this system in the NDA Drum PDP)}

\section{E. Please register the system described in this application for participation in future cycles of the NDA Drum PDP. It is understood that system tracking, test design, approval conditions, participation requirements, and audit follow-up may be based on the information supplied in this application.}

\begin{tabular}{||l|l|l||}
\hline Printed Name: & Signature: & Date: \\
\hline Phone Number: & E-Mail: & \\
\hline \hline
\end{tabular}

\section{F. DISPOSITION (coordinator use only)}




\section{Appendix D}

\section{Statistical Basis of NDA PDP Scoring Criteria}




\section{Appendix D \\ Statistical Basis of NDA PDP Scoring Criteria}

This appendix provides the basis for the NDA PDP scoring criteria. It specifies how the criteria relate to the Transuranic Waste Acceptance Criteria [WAC] for the Waste Isolation Pilot Plant (DOE/WIPP-023122). It further explains the statistical derivation of the limits and bounds criteria of the precision and bias, respectively, for the NDA PDP scoring assessment.

\section{D.1. DEFINITIONS}

\section{Limits and Bounds}

This discussion describes two types of bounds or limits: (1) those that define the acceptable precision limits for a nondestructive assay (NDA) measurement system, and (2) the allowable bias range defined by the bounds of the $95 \%$ confidence interval. While the terms "limits" and "bounds" can be used interchangeably, to avoid confusion, the term "limits" is used here only in reference to the Performance Demonstration Program (PDP) precision criteria. Similarly, the term "bounds" is used only to describe the endpoints or bounding values of calculated $95 \%$ confidence intervals for the percent recovery.

\section{Point Estimate}

A point estimate is the best single numerical value that is a good indicator of the underlying parameter of interest. Point estimates contrast with confidence bound estimates, which are interval estimates (since they delineate bounds on confidence intervals). For bias, the point estimate is the mean calculated percent recovery $(\% \mathrm{R})$, relative to the known value. For precision, the point estimate is the percent standard deviation relative to the known value $(\% \mathrm{RSD})$.

\section{D.2. PERFORMANCE CRITERIA}

For a non-interfering matrix, the NDA PDP criteria (Table D-1, column 2) specify acceptable limits for the measured precision of an NDA system based on 15 replicate assay measurements. The NDA PDP criteria are derived from the calibration confirmation criteria for NDA systems in the Transuranic Waste Acceptance Criteria [WAC] for the Waste Isolation Pilot Plant (DOE/WIPP-02-3122). The precision criterion, defined as the maximum allowable \%RSD for a non-interfering matrix and given in Table A-3.2 of the WAC, is $20.0 \%$ for 15 replicate assay measurements. This same precision limit of $20 \%$ RSD is given in Table D-1 column 2 for the lowest TRU alpha activity range.

The measured precision, based on 15 replicates, is only an approximation of the true system precision. Implicit in each limit for the measured precision is a corresponding $95 \%$ upper confidence endpoint value on the true system precision. These upper limits are stated explicitly in Table D-1. Precision criteria for NDA PDP tests for 6 replicate assay measurements, derived in relation to the upper confidence limits for 15 replicate assay measurements, are given in Table D-1 for non-interfering and interfering matrices.

The accuracy criterion for non-interfering matrix bias, as specified in the WAC, section A.3, Calibration Confirmation, is a maximum difference of $\pm 30 \%$ from $100 \%$ recovery. This criterion has been adopted for use in the NDA PDP for the non-interfering matrix bias criterion (see Table D-1). The PDP criteria for bias for the interfering matrices, shown in Table D-1, are less restrictive in consideration of the complexities involved with matrix interferences. 
Table D-1. NDA PDP Performance Criteria.

\begin{tabular}{|c|c|c|c|c|c|c|}
\hline \multirow{2}{*}{$\begin{array}{l}\text { Activity } \\
\text { Range in } \\
\alpha \text {-curies }\end{array}$} & \multirow{2}{*}{$\begin{array}{c}\text { Based on } \\
\text { WAC } \\
\text { \%RSD UL } \\
\text { for Precision } \\
\text { (15 } \\
\text { Replicates) } \\
\end{array}$} & \multirow{2}{*}{$\begin{array}{c}\text { Maximum } \\
\text { Allowable } \\
\% \mathrm{RSD} \\
\left(95 \% \mathrm{CB}^{\mathrm{b}}\right. \\
\text { of UL) }\end{array}$} & \multicolumn{2}{|c|}{$\begin{array}{c}\text { Criteria for Maximum Measured } \\
\text { PDP Precision (\%RSD) } \\
\text { (Six Replicates) }\end{array}$} & \multicolumn{2}{|c|}{$\begin{array}{c}\text { Criteria for Bias } \\
\text { (Values for } \% \mathrm{R}_{\mathrm{L}} \text { and } \% \mathrm{R}_{\mathrm{U}} \\
\text { for use in Equation } 11 \text { ) } \\
\text { (Six Replicates) }\end{array}$} \\
\hline & & & Non-interfering & Interfering & Non-interfering & Interfering \\
\hline $\begin{array}{l}>0 \text { to } \\
0.02\end{array}$ & 20 & 29.2 & 14.0 & 16 & $\begin{array}{l}\text { Lower: } 70 \\
\text { Upper: } 130\end{array}$ & $\begin{array}{l}\text { Lower: } 40 \\
\text { Upper: } 160\end{array}$ \\
\hline $\begin{array}{c}>0.02 \text { to } \\
0.2\end{array}$ & 15 & 21.9 & 10.5 & 12 & $\begin{array}{l}\text { Lower: } 70 \\
\text { Upper: } 130\end{array}$ & $\begin{array}{l}\text { Lower: } 40 \\
\text { Upper: } 160\end{array}$ \\
\hline $\begin{array}{c}>0.2 \text { to } \\
2.0\end{array}$ & 10 & 14.6 & 7.0 & 12 & $\begin{array}{l}\text { Lower: } 70 \\
\text { Upper: } 130\end{array}$ & $\begin{array}{l}\text { Lower: } 40 \\
\text { Upper: } 160\end{array}$ \\
\hline$>2.0$ & 5 & 7.3 & 3.5 & 6 & $\begin{array}{l}\text { Lower: } 70 \\
\text { Upper: } 130\end{array}$ & $\begin{array}{l}\text { Lower: } 40 \\
\text { Upper: } 160\end{array}$ \\
\hline
\end{tabular}

a - The 20\%RSD value for the low activity range was adopted from the WAC, A.3, Calibration Confirmation section. UL values for the remaining increased activity ranges were extrapolated on the basis of the capability of NDA systems of meeting these limits.

b - upper confidence bound for acceptable precision (expressed as \%RSD) at the $95 \%$ one-sided upper confidence based on 15 replicate measurements.

\section{Precision Criteria for Non-interfering Waste Matrices}

The true precision and bias of a measurement system are unknown. Estimates of the values of these parameters are acquired through the analysis of results of the total TRU $\alpha$-activity parameter from a set of six replicate assay measurements of the same NDA PDP sample. The more measurements acquired, the better are the precision and bias estimates of the NDA system for a given NDA PDP sample configuration.

The NDA PDP limits for measured precision of a non-interfering type matrix, determined from six replicate samples, are given in Table D-1. The values for six replicate measurements were derived from the upper confidence bounds for 15 replicate measurements. The derivation results in a downward adjustment of the acceptable measured precision values compared to that allowable for 15 replicate measurements, as shown in column 2.

For example, when six replicates are used, a measured value of $18 \%$ for the RSD of an assay system in the low activity range, even though less than the $20 \%$ allowable using 15 replicates, does not mean the implicit limit of an upper confidence bound of $29.2 \%$ has been met. In fact, the $95 \%$ one-sided upper confidence bound for this six-replicate example is approximately $38 \%$ - considerably higher than the allowable limit. Hence, the allowable measured precision with only six replicates is lower than that for 15 replicates for each $\alpha$-activity range.

Since the $95 \%$ confidence limit for relative standard deviation depends only on the standard deviation itself, it is possible, with a pre-specified fixed sample size, to determine ahead of time exactly how large the calculated NDA PDP precision-point estimate value can be and still have an associated upper onesided 95\% confidence limit that meets the criteria in Table D- 1 . The fourth and fifth columns of Table D-1 tabulate these maximum measured relative precision point estimate values using six replicates. The limits in columns 5 and 6 are used to compare the calculated NDA measurement system point estimate for relative standard deviation from six replicate measurements on non-interfering and interfering NDA PDP sample matrices, respectively. (Exactly how the values for interfering matrices were obtained is described below.) Note that comparing the non-interfering NDA measurement system point estimate to the value in column 4 is equivalent to comparing the associated upper one-sided $95 \%$ confidence limit to the value in column 3. That is, an NDA PDP point estimate of the value indicated in 
column 4 using six replicates will have a 95\% upper one-sided confidence limit equal to the value in column 3.

\section{Calculating Limits for Measured Relative Precision}

The limits specified in column 4 for the non-interfering PDP sample relative precision (standard deviation divided by the known value) are derived from confidence interval calculations for the variance (i.e., the square of the standard deviation) of a distribution. The derivation is described below.

\section{General Derivation}

Let $\sigma^{2}$ equal the true variance and let $1-\alpha$ equal the desired confidence value. Furthermore, let $s^{2}$ equal the sample variance, and $\chi_{\alpha, n-1}^{2}$ equal the critical value of a chi-square distribution with $n-1$ degrees of freedom above which $\alpha \%$ of the distribution lies; that is, the critical value for the upper $\alpha \%$ tail of the distribution. Then, assuming a normal distribution, a two-sided $\alpha \%$ confidence interval for the true variance is (e.g., Anderson 1987).

$$
\frac{(n-1) s^{2}}{\chi_{\alpha / 2, n-1}^{2}}<\sigma^{2}<\frac{(n-1) s^{2}}{\chi_{1-\alpha / 2, n-1}^{2}}
$$

Based on this formula for the two-sided interval, the upper one-sided $(1-\alpha) \%$ confidence limit is

$$
\sigma^{2}<\frac{(n-1) s^{2}}{\chi_{1-\alpha, n-1}^{2}}
$$

and the corresponding upper limit for the true percent relative standard deviation is calculated as

$$
\frac{\sigma}{\mu_{0}} 100 \%<\sqrt{\frac{(n-1) \frac{s^{2}}{\mu_{0}^{2}}}{\chi_{1-\alpha, n-1}^{2}}} 100 \%
$$

where $\mu_{0}$ is the reference (or true) value of the NDA PDP sample.

For the NDA PDP tests, $n=6$ and $\chi_{1-\alpha, n-1}^{2}=\chi_{0.05,5}^{2}=1.145$ in Equation D-3. Substituting these values and the known value of the NDA PDP sample for $\mu_{0}$ in this formula gives an approximate upper onesided $95 \%$ confidence limit for the percent relative standard deviation for six replicates. If desired, this upper confidence limit can be directly compared to the numbers in column 3 of Table D- 1 to determine if an assay system has met the relative precision criteria.

The numbers in column 4 of Table D-1 are derived by comparing the right portion of Equation D-3 to the appropriate number in column 3 of Table D-1 and solving for $s / \mu_{0}$. As an example, for the low activity range this calculation begins with the required inequality 


$$
\sqrt{\frac{(n-1) \frac{s^{2}}{\mu_{0}^{2}}}{\chi_{1-\alpha, n-1}^{2}}} 100 \%<29.2 \%
$$

Solving for $s / \mu_{0}$ gives

$$
\frac{s}{\mu_{0}} 100 \%<\sqrt{\frac{(0.292)^{2} \chi_{1-\alpha, n-1}^{2}}{n-1}} 100 \%
$$

which, for six samples and $95 \%$ confidence as specified in the PDP, gives

$$
\frac{s}{\mu_{0}} 100 \%<\sqrt{\frac{(0.292)^{2}(1.145)}{5}} 100 \%=14 \%
$$

Again, substituting the reference (or true) value of the NDA PDP sample for $\mu_{0}$ indicates that a calculated relative standard deviation of $14 \%$ or less meets the criterion for relative precision in the low activity range. Since the chi-square value and $n$ are the same for all activity levels, the column 4 values for the other activity levels are obtained simply by substituting the appropriate value in place of 0.292 in Equation D-6.

\section{Precision Criteria for Interfering Waste Matrices}

The WAC limits (section A.3, Calibration Confirmation) are specified for a "non-interfering matrix"; in other words, a waste matrix that does not have attributes that manifest themselves in the NDA measurement system as significant complicating error elements. To determine rational precision scoring criteria for the interfering type waste form, it was desirable to establish some relationship to program objectives that can be used as a basis for the NDA PDP criteria for the interfering matrix drums. There are certain program-defined limits for which assay systems are used to ensure compliance. In particular, there are the 200 fissile gram equivalent (FGE) material limits for 55-gallon containers and the TRU waste $\alpha$ activity definition used to discriminate TRU waste from low-level waste (LLW). At the high end, the precision of the assay system should be reasonable for waste containers approaching the 200 FGE limit to ensure that an excessive number of drums do not exceed the limit at the $95 \%$ confidence level. Similarly, the waste assay system should be sufficiently precise for containers of low TRU mass loading (i.e., in the vicinity of the $100 \mathrm{nCi} / \mathrm{gram} \alpha$-activity criterion) to ensure that an unacceptable number of containers of TRU waste are not classified as LLW.

As a convenient base for determining precision criteria for interfering type waste matrix drums, the noninterfering compliance points in Table E-1 are used. For the low activity range, the nominal compliance point for meeting the WAC precision and bias criteria is $100 \mathrm{mg}$ of weapons-grade plutonium (WG $\mathrm{Pu}$ ). An acceptable assay system should be capable of detecting and quantifying TRU waste in 55-gallon waste containers at a level of $35 \mathrm{mg} \mathrm{WG} \mathrm{Pu}$, approximately $75 \mathrm{nCi} / \mathrm{g}$ waste at 100 pounds of waste. When assaying a container at the compliance point of $100 \mathrm{mg} \mathrm{WG} \mathrm{Pu}$, we would like to be sure at the $95 \%$ confidence level that the assay system will not return a value less than $35 \mathrm{mg} \mathrm{WG} \mathrm{Pu}$. This provides reasonable protection against classifying TRU waste as LLW. Based on this rationale, two standard deviations would correspond to $65 \mathrm{mg}(100 \mathrm{mg}-35 \mathrm{mg})$. One relative standard deviation would therefore be $32.5 \mathrm{mg} / 100 \mathrm{mg}$ or 0.325 . By substituting 0.325 in place of 0.292 in Equation D-6, we obtain a value of 0.155 (rounded up to 0.16 ) for the measured precision criterion for six replicate determinations of an interfering matrix drum in the low activity range. 
Using similar reasoning, a precision criterion can be assigned to the high-mass region. In this case, the nominal compliance point used is $160 \mathrm{~g} \mathrm{WG} \mathrm{Pu}$. When assaying a container at the compliance point of $160 \mathrm{~g} \mathrm{WG} \mathrm{Pu}$, we would like to be sure at the $95 \%$ confidence level that the assay system will not return a value greater than $200 \mathrm{~g} \mathrm{WG} \mathrm{Pu}$. This provides reasonable protection against mistakenly classifying a TRU waste drum as not shippable when in fact it does not exceed the limit. Based on this rationale, two standard deviations would correspond to $40 \mathrm{~g}(200 \mathrm{~g}-160 \mathrm{~g})$. One relative standard deviation would therefore be $20 \mathrm{~g} / 160 \mathrm{~g}$ or 0.125 . By substituting 0.125 in place of 0.292 in Equation D-6, we obtain a value of 0.0598 (rounded up to 0.06 ) for the measured precision criteria for six replicate determinations of an interfering matrix drum in the high activity range.

No compelling programmatic objectives argue for specific precision limits for the low-middle and highmiddle ranges, although some thermal limits will fall into these ranges for some waste forms. Therefore, it was felt that arbitrary limits based on consistency and continuity in the use of the assay systems would be adequate for these ranges. The precision criteria for the low-middle and high-middle ranges were set at 0.12 for the RSD of six replicate determinations.

\section{D.3 CALCULATING CONFIDENCE BOUNDS FOR BIAS}

The comparison of an assay system's performance to the bias requirements for the non-interfering and interfering NDA PDP samples requires calculation of the $95 \%$ two-sided confidence bounds for the true value, using the replicate measurement data set. Based on a $t$-distribution, the $(1-\alpha) \%$ two-sided confidence bounds for the true assay system mean are (assuming a normal distribution):

$$
\bar{x}-t_{1-\alpha / 2, n-1} \frac{s}{\sqrt{n}} \leq \mu_{0} \leq \bar{x}+t_{1-\alpha / 2, n-1} \frac{s}{\sqrt{n}}
$$

In terms of percent recovery, the bounds are

$$
\frac{\bar{x}-t_{1-\alpha / 2, n-1} \frac{s}{\sqrt{n}}}{\mu_{0}} \times 100 \%<100 \%<\frac{\bar{x}+t_{1-\alpha / 2, n-1} \frac{s}{\sqrt{n}}}{\mu_{0}} \times 100 \%,
$$

where $\mu_{0}$ is the known (or true) value. The lower and upper bounds, calculated per Equation D-8, must be greater than or equal to $\% R_{L}$ and less than or equal to $\% R_{U}$, respectively, where $\% R_{L}$ and $\% R_{U}$ are the appropriate lower and upper range-specific bounds from Table D-1. Equivalently, bounds for the point estimate, total TRU $\alpha$-activity percent recovery, can be obtained by solving the required inequalities for percent recovery. The required inequalities are:

$$
\frac{\bar{x}-t_{1-\alpha / 2, n-1} \frac{s}{\sqrt{n}}}{\mu_{0}} 100 \%>\% \mathrm{R}_{\mathrm{L}} \text { and } \frac{\bar{x}+t_{1-\alpha / 2, n-1} \frac{s}{\sqrt{n}}}{\mu_{0}} 100 \% \leq \% \mathrm{R}_{\mathrm{U}}
$$

which, on solving for percent recovery, gives

$$
\% \mathrm{R}_{\mathrm{L}}+\frac{t_{1-\alpha / 2, n-1} \frac{s}{\sqrt{n}}}{\mu_{0}} 100 \% \leq \frac{\bar{x}}{\mu_{0}} 100 \% \leq \% \mathrm{R}_{\mathrm{U}}-\frac{t_{1-\alpha / 2, n-1} \frac{s}{\sqrt{n}}}{\mu_{0}} 100 \%
$$


With six samples, $n=6$, and the corresponding $t$ value (for $95 \%$ two-sided confidence bounds) is 2.571 . Thus, the equation simplifies to:

$$
\% \mathrm{R}_{\mathrm{L}}+\frac{1.049 \mathrm{~s}}{\mu_{0}} 100 \%<\frac{\bar{x}}{\mu_{0}} 100 \%<\% \mathrm{R}_{\mathrm{U}}-\frac{1.049 \mathrm{~s}}{\mu_{0}} 100 \%
$$

\section{D.4 REFERENCES}

Anderson, R. L. 1987, Practical Statistics for Analytical Chemists, New York, Van Nostrand Reinhold.

DOE/WIPP-02-3122, Transuranic Waste Acceptance Criteria for the Waste Isolation Pilot Plant, current revision, U.S. Department of Energy Waste Isolation Pilot Plant, Carlsbad, New Mexico. 


\section{Appendix E}

NDA PDP Standard Encapsulation Design 


\section{Appendix E NDA PDP Standard Encapsulation Design}

This appendix provides information on the NDA PDP standard encapsulation design used to contain the radioactive material/substrate mixture that constitutes a standard. Seven separate NDA PDP standard production phases were conducted at the Los Alamos National Laboratory. Each production phase was designed to yield a set of standards with specific attributes reflecting the DOE radioactive material inventory. All NDA PDP standards use a dual cylinder encapsulation design and have identical internal and external dimensions. The encapsulation cylinders are fabricated from seamless stainless steel tubing for all production phases except Phase III.A, which is fabricated from seamless zirconium tubing.

The PDP standard encapsulation is comprised of an inner and outer cylinder. The bottom end cap of each cylinder is laser-welded in place. The inner cylinder is then filled with the appropriate mixture of radioactive material and substrate. Once the mixture has been packed to a height specified in the production plan document (LAUR-96-2277, LAUR-98-213, LA-CP-00-54, LACP-00-110, LA-CP-01-208, LA-CP-03-0072, LA-CP-03-0763), a graphite-felt frit is installed, which presses the nuclear material/substrate assembly in place when the top cap is inserted and attached. The graphite frit also ensures that the radioactive material/substrate configuration does not change over time. The inner cylinder top cap is then welded in place using the tungsten inert gas (TIG) method. After the top cap is welded in place, the inner cylinder is inserted into the outer cylinder and the top outer cylinder cap is TIG-welded in place. An elevation view of both the inner and outer encapsulation cylinders is shown in Figure E-1. The final assembled NDA PDP standard encapsulation unit is shown in Figure E-2. Prepared assemblies of the stainless steel and zirconium dual encapsulation, complete with the substrate matrix (no radioactive material) and helium fill, have been tested in accordance with ANSI/HPS N43.6-1997 and comply with ANSI Classification 97C43323.

The dimensional and material properties of the NDA PDP standard were derived as a function of NDA PDP objectives, nondestructive waste assay system response characteristics, and practicalities of fabrication. A complete NDA PDP standard specification with supporting analyses is provided in the document, Performance Demonstration Program for Nondestructive Assay for the TRU Waste Characterization Program, Initial Cycle Source Design (INEL94/0104).

The as-specified PDP standard configuration complies with the following general requirements:

1. PDP standards must be physically stable and invariant with time in a defined geometry.

2. The PDP standard configuration must facilitate convenient loading of the standards into the PDP matrix drum or SWB.

3. The PDP standard dimensions must allow for the production of multiple-source spatial geometries within the PDP matrix drum or SWB.

4. The PDP standard encapsulation integrity must comply with all applicable standards and be acceptable for transportation to and storage at participating sites.

5. The PDP standard design must accommodate available fabrication technologies at a reasonable cost. 


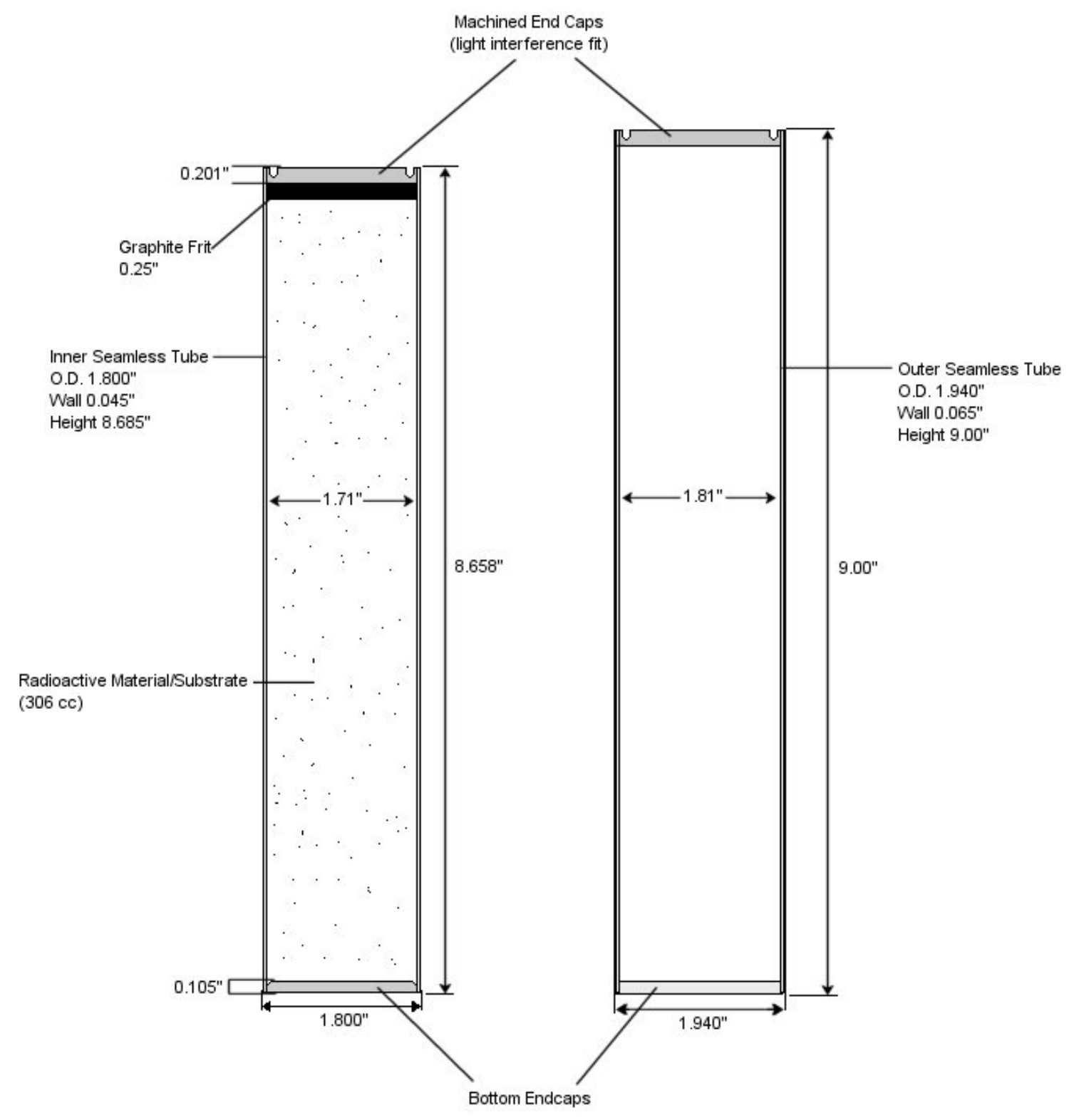

Figure E-1. PDP standard inner and outer cylinders, elevation view. 


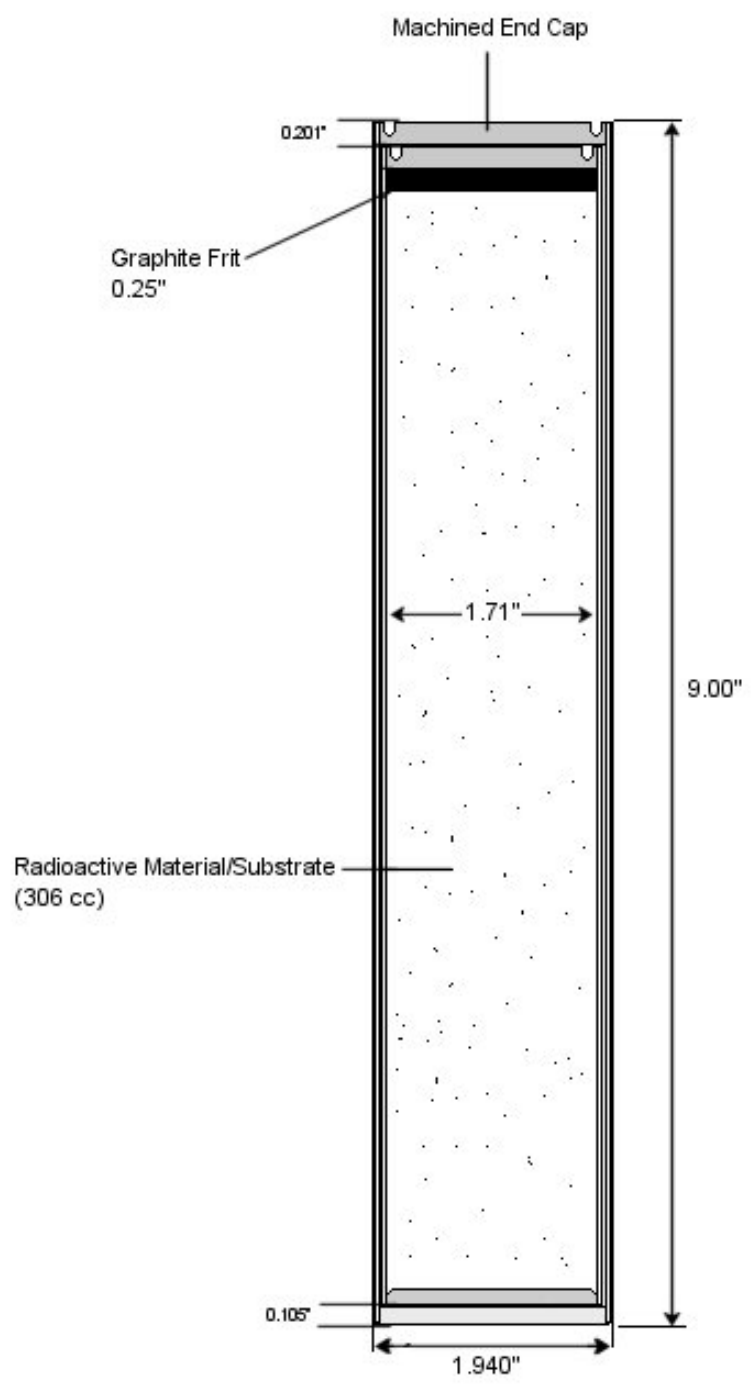

Figure E-2. PDP standard encapsulation assembly, elevation view. 


\section{Appendix F} NDA PDP Matrix Drum Specifications and Descriptions 


\section{Appendix F NDA PDP Matrix Drum Specifications and Descriptions}

This appendix provides an overview of the Nondestructive Assay (NDA) Performance Demonstration Program (PDP) matrix drum set, general specifications, design, and physical configurations. Additional technical detail on the design and fabrication of the NDA PDP matrix drum set is provided in the document Design of Interfering Matrix Drums for the Non-Destructive Waste Assay Performance Demonstration Program for the National TRU Program, INEEL/EXT-02-00320, February 2002.

Use of the waste matrix surrogate drums in combination with the NDA PDP standards provides the CBFO with test samples useful in assessing the ability of DOE facilities to meet applicable acceptance criteria for radioassay of wastes intended for disposal at the WIPP. The CBFO uses data generated in the NDA PDP as part of the assessment and approval process for measurement facilities supplying services for the characterization of WIPP TRU waste.

The waste matrix types used in the fabrication of the NDA PDP matrix drum set are based on the major DOE inventory matrix types. The basic waste categories were identified using the document Transuranic Waste Baseline Inventory Report, DOE/CAO-95-1121. These categories were subsequently collapsed based on the matrix attributes and characteristics to five waste types relative to NDA measurement technique and capability. The five waste types represented in the NDA PDP matrix drum set are: (1) noninterfering matrix (empty), (2) combustibles matrix, (3) inorganic sludge matrix, (4) metals matrix, and (5) glass matrix. Illustrations of the as-built construction of the five NDA PDP matrix drums are provided in Figures F-1 through F-5.

To position NDA PDP standard(s) within the matrix drum, insert fixtures are provided. The insert fixtures are fabricated from aluminum tubing as shown in Figure F-6. The NDA PDP standard is located at a given height within the fixture through the use of spring-loaded locating pins that snap into opposing holes in the insert fixture wall. When installed, the locating pin bisects the inner diameter of the insert fixture tube and provides a rest for the PDP standard. After the desired NDA PDP standard positions are achieved within the insert fixture, it is positioned into the matrix drum through a selected insert tube, also made of aluminum. In this manner, NDA PDP samples are configured as test samples for use in an NDA PDP cycle. The prepared NDA PDP sample must be secured with a TID to ensure the integrity of the test process and resulting data. Figure F-7 shows an NDA PDP sample with TID installed securing the insert fixtures within the matrix drum insert tubes.

General NDA PDP surrogate matrix drum specifications require the replication of the nominal waste matrix population parameters of interest, ensuring a stable non-variable matrix configuration and providing a convenient means to introduce and precisely locate PDP standards in the drum. Additionally, the NDA PDP matrix drum must conform to a set of requirements in order to support the needs of the NDA PDP Program. General specifications for an NDA PDP-type matrix drum are:

1. The design must provide a convenient means to externally introduce and locate PDP radioactive material standards (Appendix B) into the interior of a sealed 55-gallon PDP matrix drum. The design of this structure, called the internal support structure, must allow for positioning one or more PDP standards at internal drum radii and vertical heights sufficient to produce radioactivity distributions useful in performance assessments and instrument response studies. The insertion scheme and mechanism must allow for precise and reproducible positioning of standards.

2. The internal support structure used for PDP standard insertion and positioning shall also serve as a fixture allowing installed matrices to be fastened with coordinates oriented to a known reference point. 
3. The internal support structure design and materials must inherently present minimal interference to the various waste NDA techniques.

4. The surrogate matrix drum design must support the assessment of NDA system function and performance in a consistent manner over time. In accordance with this, the matrix drum must include design provisions that ensure stable, non-variable, and effectively inert matrices.

5. The surrogate waste matrix materials shall not necessitate periodic maintenance and/or inspections to validate their presence and configuration.

6. The matrix materials must be compatible with health and safety considerations such that they are not hazardous in nature, necessitating additional handling and storage precautions.

7. The selected matrix materials must be available, workable to support fabrication activities, and of reasonable cost.

8. The drum used to fabricate the surrogate matrix drum is to be of the same design and construction as used for actual packaging of DOE wastes. The drum type typically used for waste packaging purposes is a Department of Transportation (DOT) specification 17C (UN identification code UN1A2/X) 55-gallon packaging drum with a nominal 90-mil polyethylene drum liner.

9. The overall outside dimensions of the surrogate matrix drum shall be compatible with the waste NDA assay system chambers and transfer mechanisms.

10. The surrogate matrix drum must be noticeably different in appearance from actual waste drums used at the various waste characterization facilities, and from drums used for other purposes such as real-time radiography (RTR). The surrogate drum must be readily identifiable via a unique color and have a durable exterior alphanumeric identification and internal matrix code.

11. The matrix drum must be sufficiently robust in physical integrity to accommodate mechanical stresses encountered during handling and transportation. Integrity of the internal support structure and matrix must be considered in light of an expected service period of approximately 15 years.

12. The design must incorporate a means to affix an external TID for the purpose of providing control over drum contents during a measurement series. 


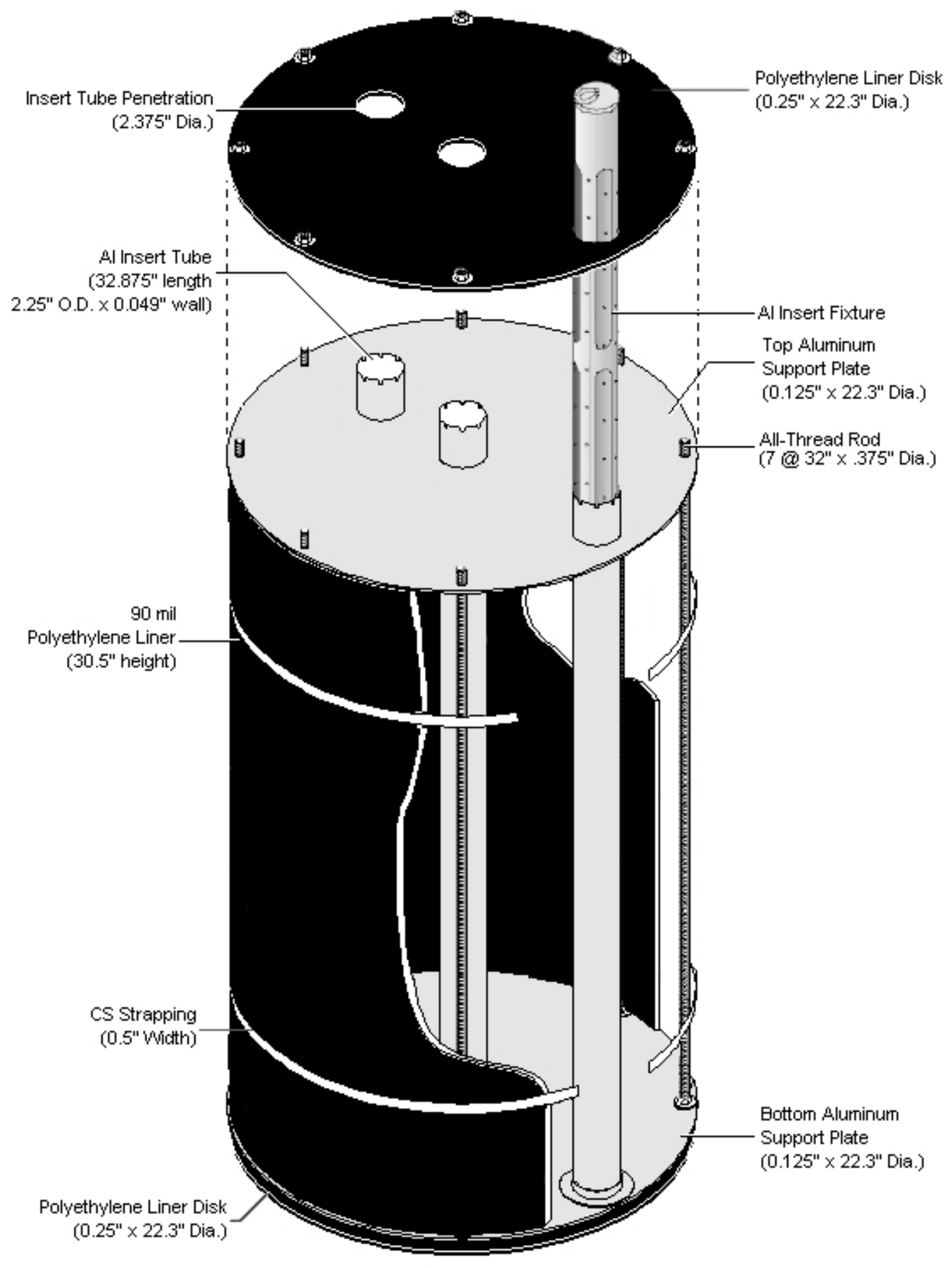

Figure F-1. NDA PDP internal support structure and non-interfering matrix drum design isometric. 


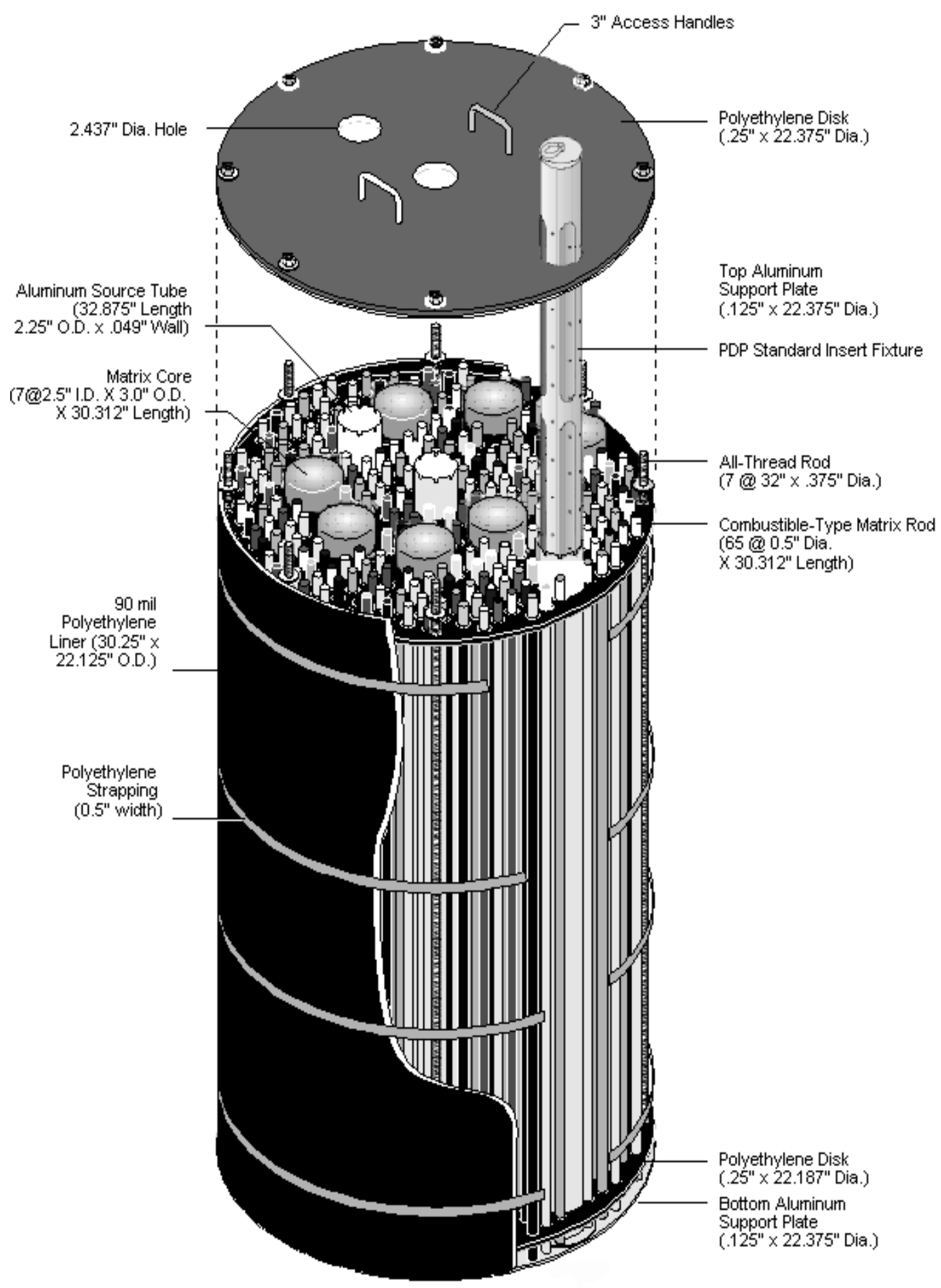

Figure F-2. NDA PDP combustibles matrix drum design isometric. 


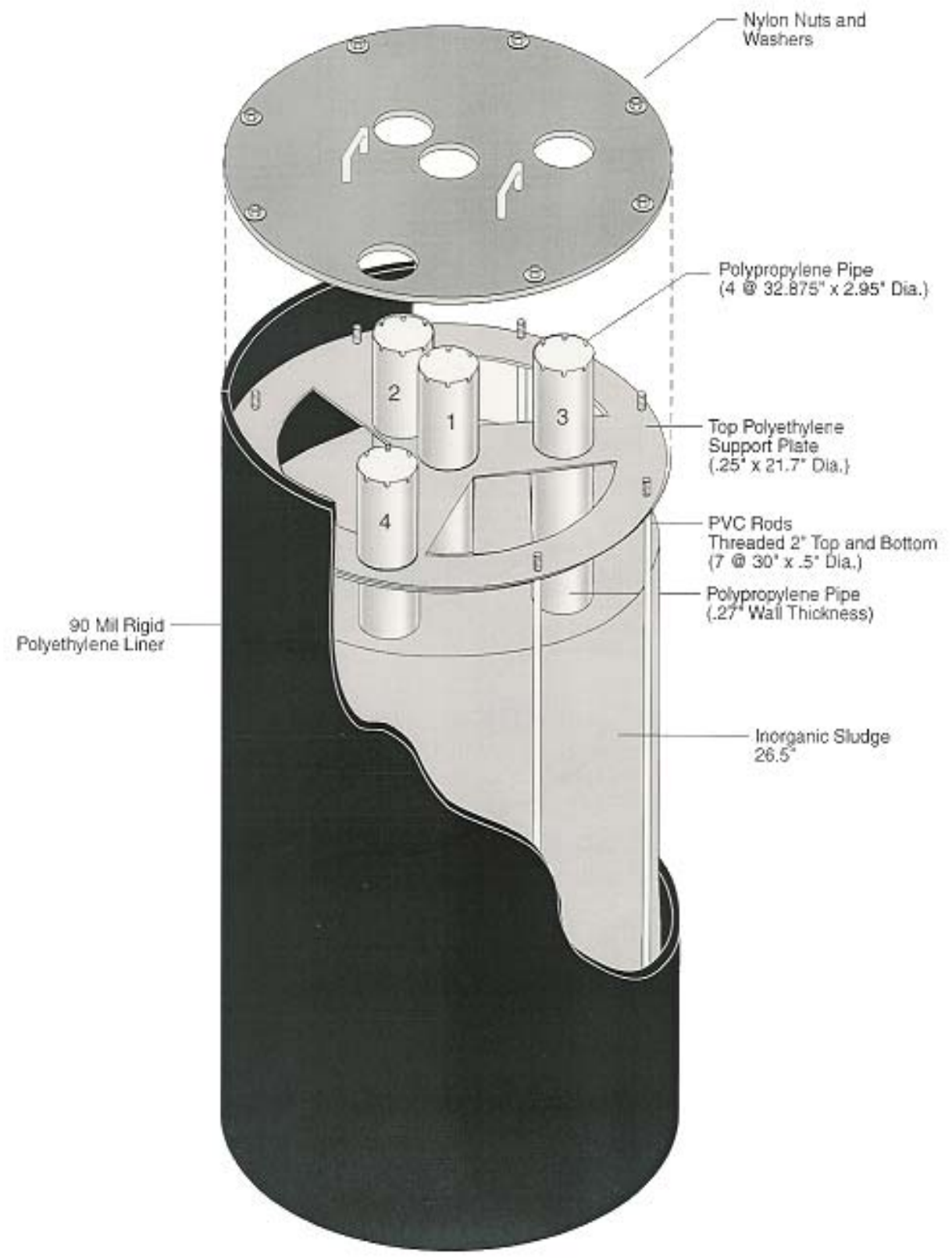

Figure F-3. NDA PDP inorganic sludge matrix drum isometric. 


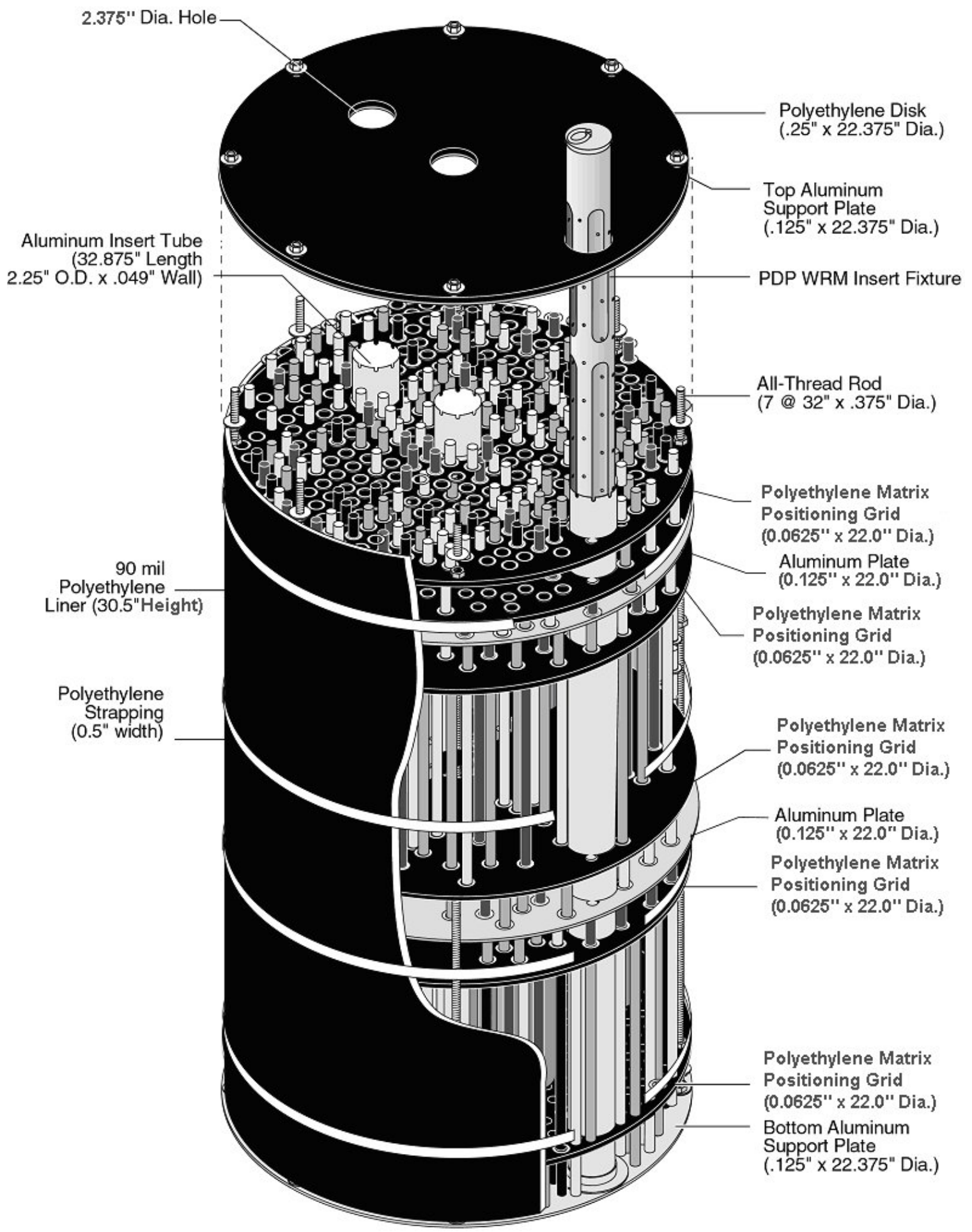

Figure F-4. NDA PDP mixed metals matrix drum isometric. 


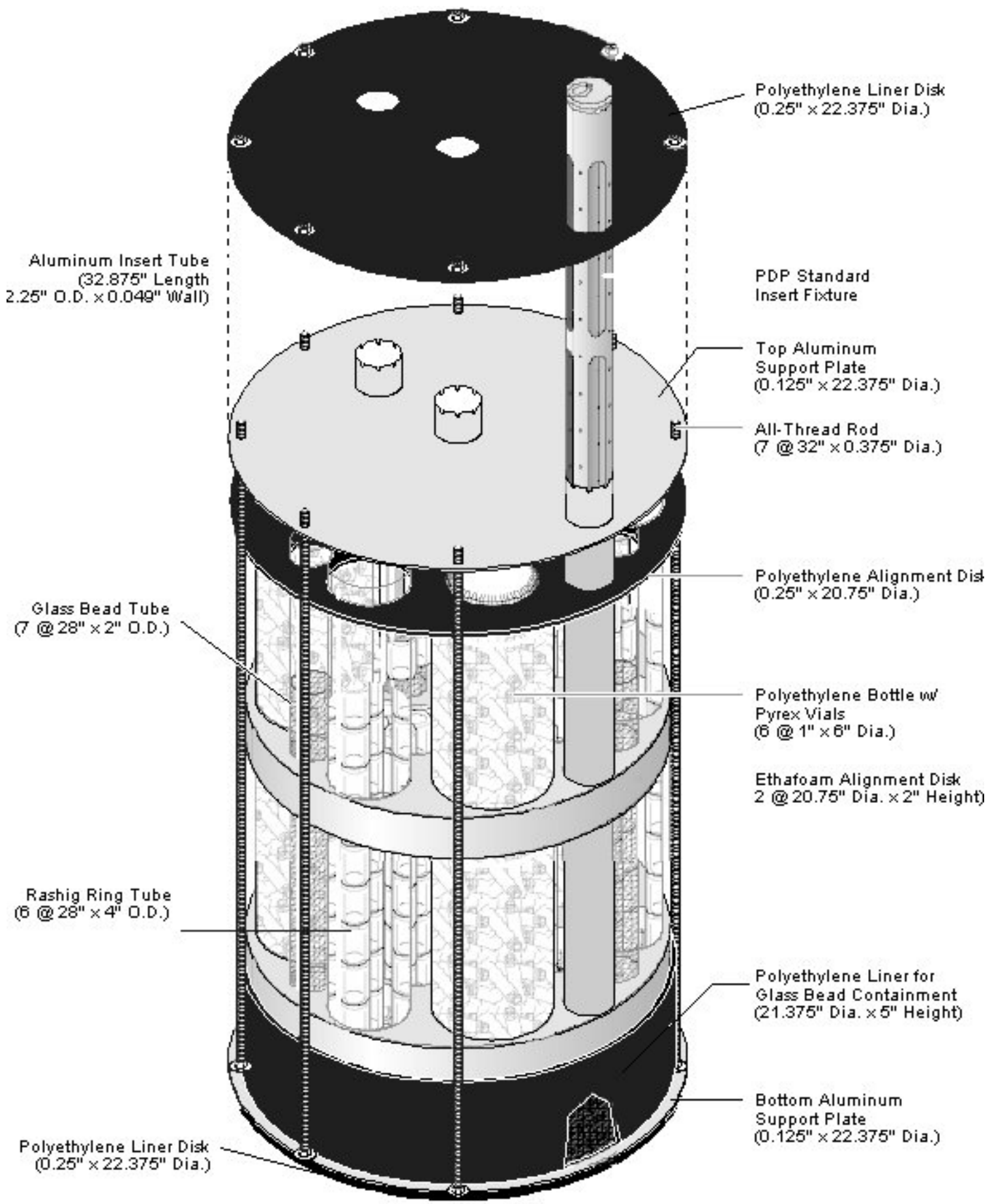

Figure F-5. NDA PDP glass matrix drum design isometric. 


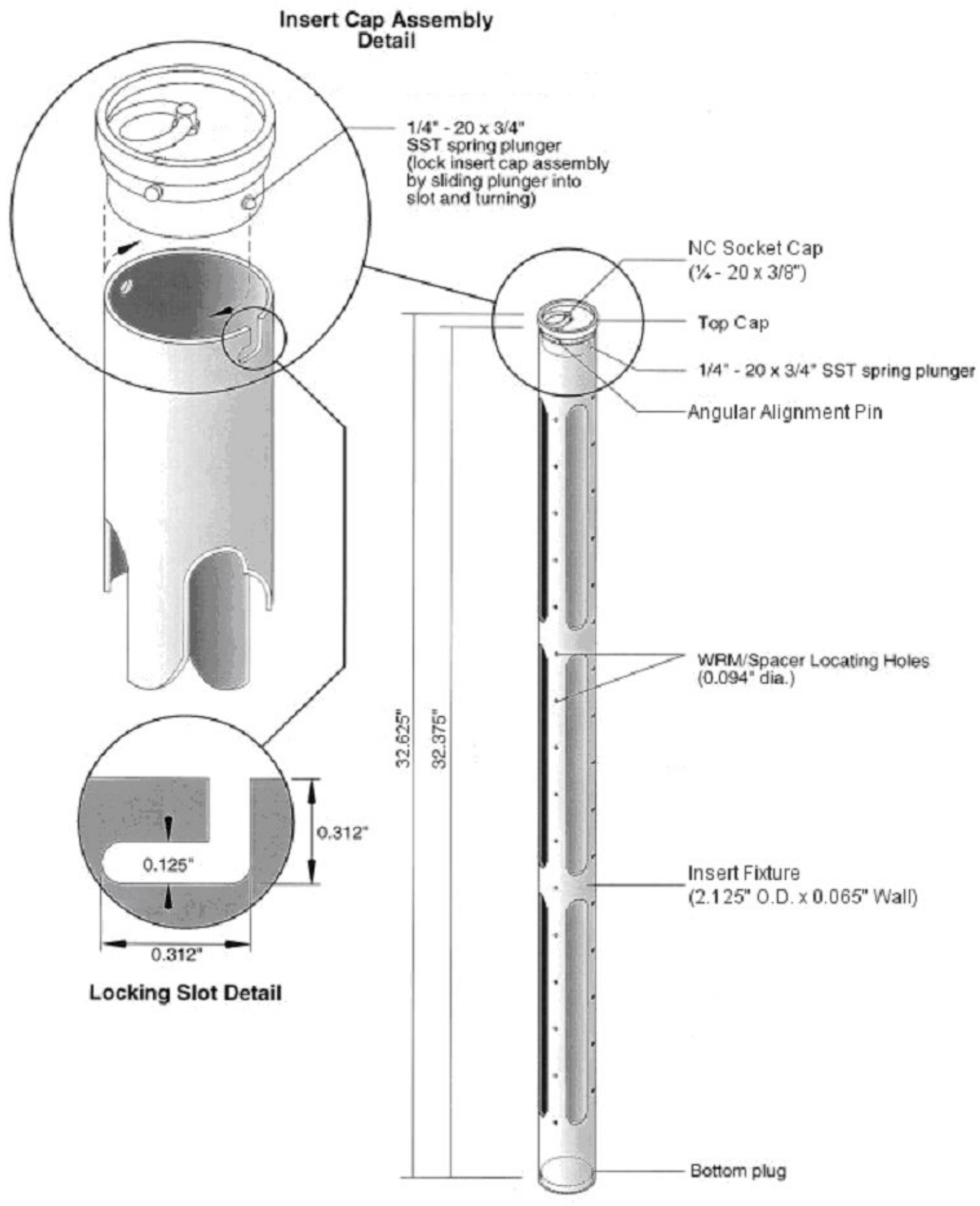

Figure F-6. NDA PDP standard insert fixture. 


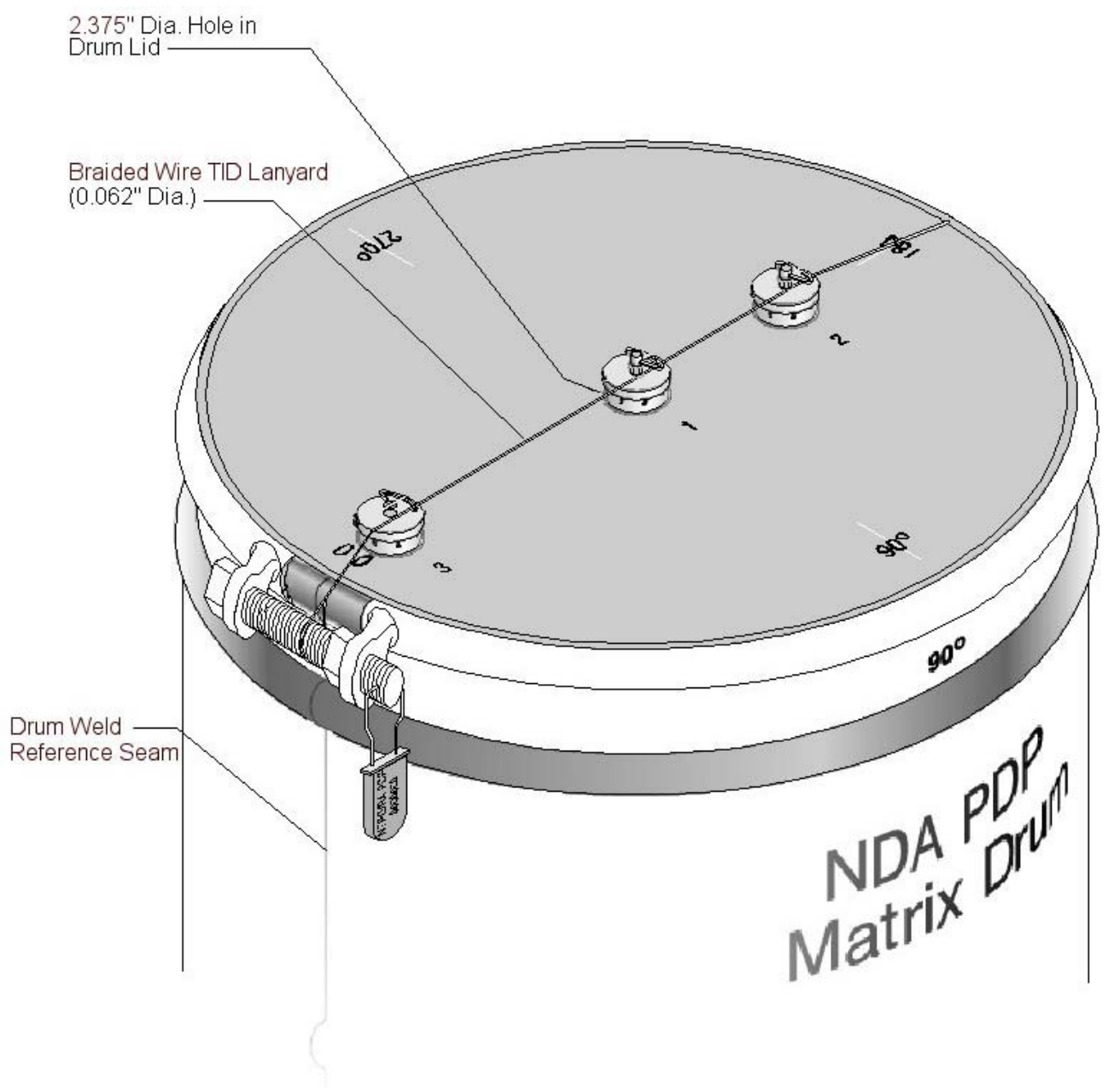

Figure F-7. NDA PDP matrix drum sample TID mechanism. 